\title{
Second-order Self-similar Identities and Multifractal Decompositions
}

\author{
KA-SING LAU \&SZE-MAN NGAI
}

ABSTRACT. Motivated by the study of convolutions of the Cantor measure, we set up a framework for computing the multifractal $L^{q}$-spectrum $\tau(q), q>0$, for certain overlapping selfsimilar measures which satisfy a family of second-order identities introduced by Strichartz et al. We apply our results to the family of iterated function systems $S_{j} x=(1 / m) x+[(m-1) m] / j$, $j=0,1, \ldots, m$, where $m$ is an odd integer, and obtain closed formulas defining $\tau(q), q>0$, for the associated self-similar measures. As a result, we can show that $\boldsymbol{\tau}(q)$ is differentiable on $(0, \infty)$ and justify the multifractal formalism in the region $q>0$. Furthermore, expressions for the Hausdorff and entropy dimensions of these measures can also be derived. By letting $m=3$, we obtain all these results for the 3-fold convolution of the standard Cantor measure.

\section{INTRODUCTION}

Let $\mu$ be a bounded positive Borel measure on $\mathbb{R}^{d}$ with compact support. For each $q \in \mathbb{R}$, define

$$
\tau(q):=\varliminf_{h \rightarrow 0^{+}} \frac{\ln \sup \sum_{i} \mu\left(B_{h}\left(x_{i}\right)\right)^{q}}{\ln h},
$$

where $\left\{B_{h}\left(x_{i}\right)\right\}$ is a family of disjoint closed $h$-balls with centers $x_{i} \in \operatorname{supp}(\mu)$, the support of $\mu$, and the supremum is taken over all such families. We call $\tau(q)$ 
the $L^{q}$-spectrum (or the moment scaling exponent) of $\mu$. It arises as an important function in the theory of multifractal measures. A heuristic principle says that the Legendre transform of $\tau(q)$, defined by $\tau^{*}(\eta):=\inf \{q \eta-\tau(q): q \in \mathbb{R}\}$, is equal to the Hausdorff dimension of the set of points whose local dimension is equal to $\eta$, i.e.,

$$
\tau^{*}(\eta)=\operatorname{dim}_{H}\left\{x \in \operatorname{supp}(\mu) \mid \lim _{h \rightarrow 0^{+}} \frac{\ln \mu\left(B_{h}(x)\right)}{\ln h}=\eta\right\} .
$$

This is known as the multifractal formalism. The right-hand side of (1.2) as a function of $\eta$ is called the dimension spectrum of $\mu$. (Here we use the letter $\eta$ instead of the more commonly used letter $\alpha$ because $\alpha$ will appear in a different context.)

Since the eighties, there have been a lot of interests and researches related to justifying the multifractal formalism and computing $\tau(q)$. We summarize some known results concerning self-similar measures, a basic class of fractal measures. Let $\left\{S_{j}\right\}_{j=0}^{m}$ be an iterated function system (IFS) of contractive similitudes of the form

$$
S_{j} x=\rho_{j} R_{j} x+b_{j}, \quad j=0,1, \ldots, m,
$$

where $0<\rho_{j}<1, R_{j}$ is an orthogonal transformation and $b_{j} \in \mathbb{R}^{d}$. For each set of probability weights $\left\{w_{j}\right\}_{j=0}^{m}$, i.e., $w_{j} \geq 0$ and $\sum_{j=0}^{m} w_{j}=1$, there corresponds a unique probability measure, called a self-similar measure, satisfying the identity

$$
\mu=\sum_{j=0}^{m} w_{j} \mu \circ S_{j}^{-1} .
$$

(See [Hut]). The multifractal formalism has been proved rigorously for self-similar or graph-directed self-similar measures satisfying the disconnected open set condition or its variants (see $[\mathrm{CM}],[\mathrm{EM}],[\mathrm{O} 1],[\mathrm{R}],[\mathrm{AP}]$ and the references therein). $\left\{S_{j}\right\}_{j=0}^{m}$ is said to satisfy the open set condition (OSC) if there exists a non-empty bounded open set $U$ such that $S_{j}(U) \subseteq U$ for all $j$ and $S_{i}(U) \cap S_{j}(U)=\varnothing$ for all $i \neq j$; and it is said to satisfy the disconnected open set condition if the last condition can be strengthened to $S_{i}(\bar{U}) \cap S_{j}(\bar{U})=\varnothing$ for all $i \neq j$. If the OSC is satisfied then $\tau(q)$ is defined by the simple equation

$$
\sum_{j=0}^{m} w_{j}^{q} \rho_{j}^{-\tau(q)}=1, \quad q \in \mathbb{R} .
$$


The OSC guarantees that $\left\{S_{j}(\operatorname{supp}(\mu))\right\}_{j=0}^{m}$ is a nonoverlapping family, in this case we also say, loosely, that the IFS $\left\{S_{j}\right\}_{j=0}^{m}$ (and the corresponding measure $\mu$ ) is nonoverlapping. If the OSC fails, we say that the IFS (and $\mu$ ) is overlapping.

In order to study some interesting overlapping cases including the well-known Bernoulli convolutions associated with the PV numbers, the authors introduced a weaker separation condition, known as the weak separation property (WSP), and justified the multifractal formalism under the assumption that $\tau^{*}(\eta)$ is strictly concave at $\eta \in \partial \boldsymbol{\tau}(q)$ where $q>0$ [LN1]. Here $\partial \boldsymbol{\tau}(q)$ is the subdifferential of $\boldsymbol{\tau}$ at $q$, defined as $\partial \tau(q)=\{\eta \mid \tau(p) \leq \tau(q)+\eta(p-q)$ for all $p \in \mathbb{R}\}$.

For such measures, one of the main unsettled problems is the calculation of $\tau(q)$. Some partial results have been obtained when $q$ is a nonnegative integer ([La1], [La2], [LN3], [FLN]). For noninteger values of $q$, much less is known. In [LN2], a closed formula defining $\tau(q), 0<q<\infty$, for the Bernoulli convolution associated with the golden ratio is derived. Recently, by using a different technique, Feng ([Fen1], [Fen2]) has obtained a formula defining $\boldsymbol{\tau}(\boldsymbol{q})$ for $q<0$ for this measure. He also obtained formulas defining $\tau(q)$ for a class of PV numbers.

The derivation of $\tau(q)$ in [LN2] is based on a set of second-order identities introduced by Strichartz et al. [STZ]. Let $\left\{S_{j}\right\}_{j=0}^{m}$ and $\mu$ be given by (1.3) and (1.4) respectively. Define

$$
T_{i} x=\rho^{n_{i}} x+d_{i}, \quad i=0,1, \ldots, \ell,
$$

where $n_{i} \in \mathbb{N}$ and $d_{i} \in \mathbb{R}^{d}$. We say that $\mu$ satisfies a family of second-order self-similar identities (or simply second-order identities) with respect to $\left\{T_{i}\right\}_{i=0}^{\ell}$ if

(i) $\operatorname{supp}(\mu) \subseteq \bigcup_{i=0}^{\ell} T_{i}(\operatorname{supp}(\mu))$ and

(ii) for each $A \subseteq \operatorname{supp}(\mu)$ and $0 \leq i, j \leq \ell, \mu\left(T_{i} T_{j} A\right)$ can be expressed as a linear combination of $\left\{\mu\left(T_{k} A\right) \mid k=0,1, \ldots, m\right\}$ as

$$
\mu\left(T_{i} T_{j} A\right)=\sum_{k=0}^{m} c_{k} \mu\left(T_{k} A\right),
$$

where $c_{k}=c_{k}(i, j)$ are independent of $A$. (Here $T_{i} T_{j}$ denotes the composition $T_{i} \circ T_{j}$.)

For our purposes, $\left\{T_{i}\right\}_{i=0}^{\ell}$ needs to satisfy the OSC.

Second-order identities were introduced in [STZ] to compute numerical approximations to the measure. If $\left\{S_{j}\right\}_{j=0}^{m}$ satisfies the OSC with an open set $U$, then using a theorem of Schief [S, Theorem 2.2] we can assume that $\mu(U)=1$. Hence for all $A \subseteq \operatorname{supp}(\mu), \mu\left(S_{i} S_{j}(A)\right)=w_{i} \mu\left(S_{j}(A)\right)$ and therefore $\mu$ satisfies a family of second-order identities with respect to $\left\{S_{j}\right\}_{j=0}^{m}$ itself. 
The formula for $\boldsymbol{\tau}(q)$ derived in [LN2] yields important and new information for the infinite Bernoulli convolution associated with the golden ratio, which has been studied extensively (see $[\mathrm{AY}],[\mathrm{AZ}],[\mathrm{Hu}],[\mathrm{L}],[\mathrm{LP}],[\mathrm{P}])$. These include the differentiability of $\boldsymbol{\tau}(q)$ and the dimension spectrum of the measure for $q>0$, a formula for its Hausdorff and entropy dimensions, and formulas for its $L^{q_{-}}$ dimensions and its $L^{\infty}$-dimension. Unfortunately, the method in [LN2] cannot be applied to measures associated with other well-known PV numbers because they do not satisfy similar second-order identities. It is the purpose of this paper to carry out a systematic study of this method and show that it does generalize to some other classes of overlapping measures, including the interesting 3-fold convolution of the Cantor measure.

We consider equicontractive similitudes of the form

$$
S_{j} x=\rho x+b_{j}, \quad j=0,1, \ldots, m,
$$

where $0<\rho<1$ and $0=b_{0}<b_{1}<\cdots<b_{m}$. We are interested in the case $\left\{S_{j}\right\}_{j=0}^{m}$ does not satisfy the OSC. Let $\mu$ be the corresponding self-similar measure. We will prove that if $\rho=1 / k$, where $k \geq 2$ is an integer, $b_{j}=[(k-1) / k] j$ for $j=0,1, \ldots, m$, and $m=k^{N}$ for some $N \in \mathbb{N}$, then we can define

$$
T_{i} x=\frac{1}{k^{N}} x+i, \quad i=0,1, \ldots, m-1,
$$

so that with respect to $\left\{T_{i}\right\}_{i=0}^{m-1}, \mu$ satisfies a family of second-order identities.

Our main objective is to make use of the second-order identities to formulate a set of conditions under which a closed formula defining $\tau(q)$ can be derived. Note that $\operatorname{supp}(\mu) \subseteq[0, a]$, where $a=b_{m} /(1-\rho)$. Define

$$
T_{i} x=\rho^{n_{i}} x+d_{i}, \quad d_{i}=0,1, \ldots, \ell,
$$

where $n_{i}$ is a positive integer, and suppose $\left\{T_{i}\right\}_{i=0}^{m-1}$ is a nonoverlapping family with respect to which $\mu$ satisfies a family of second-order identities. For the purposes of this paper we only consider the case in which $\left\{T_{i}\right\}_{i=0}^{\ell}$ can be partitioned into two subfamilies $\left\{T_{i}\right\}_{i \in \mathcal{I}_{0}}$ and $\left\{T_{i}\right\}_{i \in \mathcal{I}_{1}}$, each is equicontractive with contraction ratios $\rho^{n}$ and $\rho^{\tilde{n}}$ respectively.

The derivation of $\boldsymbol{\tau}(q)$ is based on the following well-known equivalent definition, which holds for $q>0$ :

$$
\tau(q)=\inf \left\{\alpha \mid \varlimsup_{h \rightarrow 0^{+}} \frac{1}{h^{1+\alpha}} \int_{-\infty}^{\infty} \mu\left(B_{h}(x)\right)^{q} d x>0\right\} .
$$


Let

$$
\Phi^{(\alpha)}(h):=\frac{1}{h^{1+\alpha}} \sum_{i \in I_{1}} \int_{0}^{a} \mu\left(B_{h}\left(T_{i} x\right)\right)^{q} d x
$$

Under certain additional conditions on the similitudes and the second-order identities (see (C1), (C2) and (C3) in Section 3), $\Phi^{(\alpha)}(h)$ behaves like $1 / h^{1+\alpha} \int_{0}^{a} \mu\left(B_{h}(t)\right)^{q} d t$ as $h \rightarrow 0^{+}$and the following functional equation for $\Phi^{(\alpha)}(h)$ can be derived:

$$
\begin{aligned}
\Phi^{(\alpha)}(h)=\sum_{k=1}^{t-1} \rho^{-((k-1) n+\tilde{n}) \alpha} c_{k}^{0}(q) \Phi^{(\alpha)}\left(\frac{h}{\rho^{(k-1) n+\tilde{n}}}\right) \\
+\sum_{k=1}^{t-1} \rho^{-k n \alpha} c_{k}^{1}(q) \Phi^{(\alpha)}\left(\frac{h}{\rho^{k n}}\right) \\
+\sum_{k=0}^{N} \rho^{-(t n+k \tilde{n}) \alpha} \widetilde{c}_{k}(q) \Phi^{(\alpha)}\left(\frac{h}{\rho^{t n+k \tilde{n}}}\right)+E(h),
\end{aligned}
$$

where $t$ is some fixed positive integer, $c_{k}^{0}(q), c_{k}^{1}(q), \widetilde{c}_{k}(q)$ are functions of $q, N$ is the largest integer satisfying $0<h \leq \rho^{(t+1) n+N \tilde{n}}$, and $E(h)$ is some error term.

Once (1.11) is established, the formula for $\tau(q)$ follows from the renewal theorem. More precisely, define

$$
\begin{aligned}
F(q, \alpha) & :=\sum_{k=1}^{t-1}\left(\rho^{-((k-1) n+\tilde{n}) \alpha} c_{k}^{0}(q)+\rho^{-k n \alpha} c_{k}^{1}(q)\right)+\sum_{k=0}^{\infty} \rho^{-(t n+k \tilde{n}) \alpha} \widetilde{\mathcal{C}}_{k}(q) \\
D & :=\{(q, \alpha) \mid q>0, F(q, \alpha)<\infty\} .
\end{aligned}
$$

For $q>0$, let $\tilde{\alpha}=\widetilde{\alpha}(q)$ be the unique $\alpha$ such that $(q, \alpha) \in \partial D$, the boundary of $D$. We have the following main result:

Theorem 1.1. Suppose that for each $q>0, F(q, \alpha)$ tends to $\infty$ as $\alpha$ increases to $\widetilde{\alpha}$, and suppose that for $\alpha$ satisfying $F(q, \alpha)=1$, there exists $\varepsilon>0$ such that $E(h)=o\left(h^{\varepsilon}\right)$ as $h \rightarrow 0^{+}$. Then $\tau(q)=\alpha$, where $\alpha$ is the unique solution of $F(q, \alpha)=1$. Moreover, $\tau$ is differentiable on $(0, \infty)$.

The differentiability of $\tau$ on $(0, \infty)$ is important because it implies that $\tau^{*}(\eta)$ is strictly concave for all $\eta \in \partial \tau(q), q>0$, and hence for such $\eta$ the multifractal formalism (i.e., equality (1.2)) holds [LN1]. 
$\tau(q)$ is intimately related to several other notions of dimension of the measure $\mu$. For $q>1$, the (lower) $L^{q}$-dimension (or generalized Rényi dimension) ([St], [HP]) of $\mu$, denoted by $\underline{\operatorname{dim}}_{q}(\mu)$, can be defined as

$$
\underline{\operatorname{dim}}_{q}(\mu)=\frac{\tau(q)}{q-1}, \quad q>1 .
$$

For $q=\infty$ we define the lower $L^{\infty}$-dimension of $\mu$ as

$$
\underline{\operatorname{dim}}_{\infty}(\mu)=\lim _{h \rightarrow 0^{+}} \frac{\ln \sup \mu\left(B_{h}(x)\right)}{\ln h},
$$

where $x \in \operatorname{supp}(\mu)$ and the supremum is taken over all such $x$ (see [LN1], [St]). $\underline{\operatorname{dim}}_{\infty}(\mu)$ corresponds to the left end-point of the dimension spectrum.

$\tau(q)$ is also related to the Hausdorff and entropy dimensions of $\mu$. Assume now that $v$ is a Borel probability measure on $\mathbb{R}^{d}$ with bounded support (not necessarily a self-similar measure). For a finite Borel partition $\mathcal{P}$ of $\operatorname{supp}(v)$, let $|\mathcal{P}|$ be the maximum of the diameters of elements of $\mathcal{P}$. Define

$$
H(\nu, \mathcal{P})=-\sum_{A \in \mathcal{P}} v(A) \ln v(A),
$$

and for $h>0$, let

$$
H(\nu, h)=\inf \{H(\nu, \mathcal{P}) \mid \mathcal{P} \text { is a finite Borel partition of } \operatorname{supp}(\nu),|\mathcal{P}| \leq h\} .
$$

The entropy dimension of $v$ is defined as

$$
\operatorname{dim}_{e}(v)=\lim _{h \rightarrow 0^{+}} \frac{H(\nu, h)}{-\ln h} .
$$

The Hausdorff dimension of $v$ is defined as

$$
\operatorname{dim}_{H}(v)=\inf \left\{\operatorname{dim}_{H}(E) \mid v\left(\mathbb{R}^{d} \backslash E\right)=0\right\} .
$$

It is proved by one of the authors in [N2, Theorem 1.1] that, if $\tau(q)$ is differentiable at $q=1$, then $\operatorname{dim}_{H}(v)=\operatorname{dim}_{e}(v)=\tau^{\prime}(1)$ (see also generalizations by Heurteaux $[\mathrm{H}]$ and Olsen [O2]). In this case we will call the common value the dimension of $v$ and denote it by $\operatorname{dim}(v)$.

Under the hypotheses of Theorem 1.1, we immediately obtain formulas for $\underline{\operatorname{dim}}_{q}(\mu)$ and $\underline{\operatorname{dim}}_{\infty}(\mu)$. Moreover, since the differentiability of $\tau$ for $q>0$ is guaranteed, we can hence derive a closed formula for $\operatorname{dim}(\mu)$ (see Corollary 3.3). 
We apply Theorem 1.1 to the measures defined by

$$
S_{j} x=\frac{1}{m} x+\frac{m-1}{m} j, \quad j=0,1, \ldots, m,
$$

where $m$ is an "odd" integer. A corresponding collection of $T_{i}$ is

$$
T_{i} x=\frac{1}{m} x+i, \quad i=0,1, \ldots, m-1 .
$$

In this case $\mathcal{I}_{0}=\{0, m-1\}, \mathcal{I}_{1}=\{1, \ldots, m-2\}$, and $F(q, \alpha)$ is simplified to

$$
F(q, \alpha)=m^{\alpha} \sum_{i=1}^{m-1} w_{i}^{q}+\sum_{k=0}^{\infty} m^{(k+2) \alpha}\left(\sum_{i=1}^{m-2} \sum_{|J|=k} c_{i, J}^{q}\right),
$$

where $J=\left(j_{1}, \ldots, j_{k}\right), j_{i}=0$ or $m-1$, and $|J|=k$ denotes the length of $J . c_{i, J}$ is defined as

$$
c_{i, J}=\left[w_{i+1}, w_{i}\right] P_{J}\left[\begin{array}{c}
w_{0} \\
w_{m}
\end{array}\right],
$$

where $P_{J}$ is the matrix product $P_{j_{1}} \cdots P_{j_{k}}$ and

$$
P_{0}=\left[\begin{array}{cc}
w_{0} & 0 \\
w_{m} & w_{m-1}
\end{array}\right], \quad P_{m-1}=\left[\begin{array}{cc}
w_{1} & w_{0} \\
0 & w_{m}
\end{array}\right] .
$$

We have the following main result concerning this family of measures.

Theorem 1.2. Let $m \geq 3$ be an odd integer and let $S_{j}(x)=(1 / m) x+$ $[(m-1) / m] j, j=0,1, \ldots, m$. Let $\mu$ be the self-similar measure defined by $\left\{S_{j}\right\}_{j=0}^{m}$ together with a set of nonnegative weights $\left\{w_{j}\right\}_{j=0}^{m}$. Suppose that $w_{0}, w_{m}>0$ and that there exists $1 \leq j_{0} \leq m-1$ such that $w_{j_{0}}>0$. Then $\tau(q)=\alpha$, where $\alpha$ is defined by

$$
m^{\alpha} \sum_{i=1}^{m-1} w_{i}^{q}+\sum_{k=0}^{\infty} m^{(k+2) \alpha}\left(\sum_{i=1}^{m-2} \sum_{|J|=k} c_{i, J}^{q}\right)=1 .
$$

Moreover, $\tau$ is differentiable on $(0, \infty)$.

Corollary 1.3. For the measure $\mu$ in Theorem 1.2, the multifractal formalism holds for $q>0$, i.e.,

$$
\tau^{*}(\eta)=\operatorname{dim}_{H}\left\{x \in \operatorname{supp}(\mu) \mid \lim _{h \rightarrow 0^{+}} \frac{\ln \mu\left(B_{h}(x)\right)}{\ln h}=\eta\right\},
$$

where $\eta=\tau^{\prime}(q)$ and $q>0$. 
We are not able to get similar results if $m$ is even, although analogous secondorder identities exist. It is not clear how a suitable collection of $T_{i}$ can be defined so that an analogue of the functional equation (1.11) can be derived.

Our present work is motivated by the study of the 3-fold convolution of the standard Cantor measure. Unlike the standard Cantor measure or its 2-fold convolution, the 3 -fold convolution is defined by the following set of similitudes that fails to satisfy the OSC:

$$
S_{j} x=\frac{1}{3} x+\frac{2}{3} j, \quad j=0,1,2,3 .
$$

The corresponding measure $\mu$ is defined by

$$
\mu=\frac{1}{8} \mu \circ S_{0}^{-1}+\frac{3}{8} \mu \circ S_{1}^{-1}+\frac{3}{8} \mu \circ S_{2}^{-1}+\frac{1}{8} \mu \circ S_{3}^{-1} .
$$

In [FLN], it is shown that the $S_{j}$ in (1.18) satisfy the WSP and a matrix method is used to compute $\tau(q)$ when $q$ is equal to a nonnegative integer. By using the results in this paper, we can obtain the following formula defining $\tau(q)$ for $q>0$ and show that $\tau(q)$ is differentiable on $(0, \infty)$. The matrices $P_{0}$ and $P_{2}$ in (1.17) become

$$
P_{0}=\frac{1}{8}\left[\begin{array}{ll}
1 & 0 \\
1 & 3
\end{array}\right] \text { and } P_{2}=\frac{1}{8}\left[\begin{array}{ll}
3 & 1 \\
0 & 1
\end{array}\right] .
$$

Let $c_{J}=\frac{3}{64}[1,1] P_{J}\left[\begin{array}{l}1 \\ 1\end{array}\right]$. Then we have the following result:

Theorem 1.4. Let $\mu$ be the 3-fold convolution of the standard Cantor measure. Then

(a) $\tau(q)=\alpha$, with $\alpha$ defined by

$$
2 \cdot 3^{\alpha}\left(\frac{3}{8}\right)^{q}+\sum_{k=0}^{\infty} 3^{(k+2) \alpha}\left(\sum_{|J|=k} c_{J}^{q}\right)=1,
$$

where $J=\left(j_{1}, \ldots, j_{k}\right), j_{i}=0$ or 2. Moreover, $\boldsymbol{\tau}(q)$ is differentiable on $(0, \infty)$.

(b)

$$
\text { (1.20) } \operatorname{dim}(\mu)=\tau^{\prime}(1)=\frac{9 \ln (3 / 8)+12 \sum_{k=0}^{\infty} \sum_{|J|=k} c_{J} \ln c_{J}}{-20 \ln 3} \approx 0.9884 \ldots .
$$


(c) $\underline{\operatorname{dim}}_{\infty}(\mu)=\ln \frac{8}{3} / \ln 3(\approx 0.89278926 \ldots)$.

We have not investigated the calculation of $\tau(q)$ for $q<0$; the reader is referred to some related results by $\mathrm{Hu}$ and $\mathrm{Lau}[\mathrm{HL}]$.

This paper is organized this as follows. In Section 2, we study some basic properties and examples of second-order identities. In Section 3 we derive the key functional equation (1.11) and prove Theorem 1.1. In Section 4, we apply our results to the IFS in (1.13) and derive Theorem 1.2 and Corollary 1.3. In Section 5 , we simplify the formula for $\tau(q)$ in the case $q$ is a positive integer. In Section 6 , we study the 3 -fold convolution of the Cantor measure and prove Theorem 1.4. The proof of Theorem 1.2 involves a technical estimation of the error term $E(h)$ stated in Theorem 1.1. Some techniques have already been used in [LN2]; we include the estimations in the Appendix for completeness.

\section{SECOND-ORDER IDENTITIES}

In this section we study some properties and examples concerning second-order identities. Let $k \geq 2$ be an integer, let

$$
S_{j} x=\frac{1}{k} x+\frac{k-1}{k} j, \quad j=0,1, \ldots, m,
$$

and let $\mu$ be the self-similar measure defined by the $S_{j}$ as in (1.4). We remark that for any $r \in \mathbb{R}$, the self-similar measure defined by the similitudes $\widetilde{S_{j}} x=$ $(1 / k) x+r j, j=0,1, \ldots, m$, together with the same set of weights $\left\{w_{j}\right\}_{j=0}^{m}$, can be identified with $\mu$. It can also be checked that $\operatorname{supp}(\mu) \subseteq[0, m]$ and $\left\{S_{j}\right\}_{j=0}^{m}$ satisfies the OSC if and only if $m \leq k-1$.

Now assume that $m=k^{N}$ for some $N \in \mathbb{N}$. Note that in this case the OSC fails. We define

$$
T_{i} x=\frac{1}{k^{N}} x+i, \quad i=0,1, \ldots, m-1 .
$$

Since $T_{i}[0, m]=[i, i+1],\left\{T_{i}\right\}_{i=0}^{m-1}$ satisfies the OSC with $(0, m)$ as an open set. For any multi-index $I=\left(i_{1}, \ldots, i_{k}\right)$, we denote the composition $T_{i_{1}} \circ \cdots \circ T_{i_{k}}$ by $T_{i_{1}} \cdots T_{i_{k}}$ or $T_{I}$, and the composition $S_{i_{1}}^{-1} \circ \cdots \circ S_{i_{k}}^{-1}$ by $S_{i_{1}}^{-1} \cdots S_{i_{k}}^{-1}$.

Proposition 2.1. Let $\mu$ be a self-similar measure defined by the similitudes in (2.1) and let $m=k^{N}$ for some integer $N$. Then $\mu$ satisfies a set of second-order identities with respect to the maps $T_{i}$ in (2.2). 
Proof. Let $A \subseteq[0, m]$. By applying (1.4) repeatedly, we have for $0 \leq i$, $j \leq m-1$,

$$
\begin{aligned}
\mu\left(T_{i} T_{j} A\right) & =\sum_{\ell_{1}, \ldots, \ell_{N}=0}^{m} w_{\ell_{1}} \cdots w_{\ell_{N}} \mu\left(S_{\ell_{N}}^{-1} \cdots S_{\ell_{1}}^{-1}\left(\frac{1}{k^{2 N}} A+\frac{1}{k^{N}} j+i\right)\right) \\
& =\sum_{\ell_{1}, \ldots, \ell_{N}=0}^{m} w_{\ell_{1}} \cdots w_{\ell_{N}} \mu\left(\frac{1}{k^{N}} A+s\right),
\end{aligned}
$$

where $s=s\left(i, j, \ell_{1}, \ldots, \ell_{N}\right):=j+k^{N} i-(k-1) \sum_{r=1}^{N} k^{N-r} \ell_{r}$. Since $\operatorname{supp}(\mu) \subseteq$ $[0, m]$ and $s$ can take only integer values, $\mu\left[\left(1 / k^{N}\right) A+s\right] \neq 0$ if and only if $s=0,1, \ldots, m-1$. Hence $\mu\left(T_{i} T_{j} A\right)$ is a linear combination of $\left\{\mu\left(T_{k} A\right) \mid k=\right.$ $0,1, \ldots, m-1\}$.

Example. Putting $N=1$, we get the following example

$$
S_{j} x=\frac{1}{m} x+\frac{m-1}{m} j, \quad j=0,1, \ldots, m .
$$

In this case we can define

$$
T_{i} x=\frac{1}{m} x+i, \quad i=0,1, \ldots, m-1 .
$$

We will come back to this family in Section 4.

Remark. The $\left\{T_{i}\right\}_{i=0}^{m-1}$ in (2.2) is not the unique family with respect to which $\mu$ satisfies a set of second-order identities. In fact, if $m=k^{N}$ then for each $n \in \mathbb{N}$ we can define

$$
\tilde{T}_{i} x=\frac{1}{k^{n} k^{N}} x+\frac{i}{k^{n}}, \quad i=0,1, \ldots, m k^{n}-1 .
$$

The proof of Proposition 2.1 shows that the same conclusion holds for the maps $\left\{\tilde{T}_{i}\right\}_{i=0}^{m k^{n}-1}$. The proof of Proposition 2.1 also yields the following important matrix identities, which hold for all $A \subseteq[0, m]$ :

$$
\left[\begin{array}{c}
\mu\left(T_{0} T_{i} A\right) \\
\mu\left(T_{1} T_{i} A\right) \\
\vdots \\
\mu\left(T_{m-1} T_{i} A\right)
\end{array}\right]=M_{i}\left[\begin{array}{c}
\mu\left(T_{0} A\right) \\
\mu\left(T_{1} A\right) \\
\vdots \\
\mu\left(T_{m-1} A\right)
\end{array}\right], \quad i=0,1, \ldots, m-1,
$$


where $M_{i}$ is some $m \times m$ matrix. Let $J=\left(j_{1}, \ldots, j_{k}\right), j_{i}=1, \ldots, m-1$, and let $\mathbf{e}_{i}$ denote the unit vector in $\mathbb{R}^{m}$ whose $(i+1)$-st coordinate is 1 . Applying (2.3) repeatedly yields

$$
\mu\left(T_{J}[0, m]\right)=\mathbf{e}_{j_{1}}^{T} M_{j_{2}} \cdots M_{j_{k}}\left[\begin{array}{c}
\mu\left(T_{0}[0, m]\right) \\
\mu\left(T_{1}[0, m]\right) \\
\vdots \\
\mu\left(T_{m-1}[0, m]\right)
\end{array}\right]:=d_{J} .
$$

Using (2.4) we obtain the following uniqueness result for the $\mu$ satisfying (2.3).

Proposition 2.2. The system of second-order identities in (2.3) together with the values $\mu\left(T_{i}[0, m]\right), i=0,1, \ldots, m-1$ uniquely determines a Borel probability measure on $[0, m]$.

Proof. We see from (2.4) that for all $J=\left(j_{1}, \ldots, j_{k}\right)$ with $j_{i}=0,1, \ldots$, $m-1$, the values $\mu\left(T_{J}[0, m)\right)$ are uniquely determined by the matrices $M_{i}$ and the values $\mu\left(T_{i}[0, m]\right), i=0,1, \ldots, m-1$. Hence $\mu$ is uniquely determined on each of the $m$-adic intervals $\left\{T_{J}[0, m)|| J \mid=k\right\}$. The assertion now follows from Carathéodory's extension theorem [Ro].

Write $\rho=1 / k^{N}$. We have the following formula, which can be used to approximate $\tau(q)$ for "all" $q \in \mathbb{R}$. The approximations are better for smaller absolute values of $q$.

Theorem 2.3. Assume the same hypotheses of Proposition 2.1 and assume that the probability weights satisfy $w_{j}>0$ for all $j$. Then for all $q \in \mathbb{R}$,

$$
\tau(q)=\lim _{n \rightarrow \infty} \frac{\ln \sum_{|J|=n} d_{j}^{q}}{n \ln \rho},
$$

where $\rho=1 / k^{N}$ and $d_{J}$ is defined by (2.4).

Proof. We first remark that, since all $w_{j}$ are positive, $\mu$ does not have point masses and $\operatorname{supp}(\mu)=[0, m]$. The proof for $q>0$ follows from an obvious generalization of that in [N1, Theorem 2.4]. Now assume $q<0$. Since $\mu$ does not have point masses, the closed ball $B_{h}\left(x_{i}\right)$ in the definition of $\tau(q)$ can be 
replaced by the open ball $B_{h}^{\circ}\left(x_{i}\right)$. By regarding $\left\{T_{J}(0, m)\right\}_{|J|=n}$ as a family of disjoint open $\left(m \rho^{n} / 2\right)$-balls with centers in $\operatorname{supp}(\mu)$, we have

$$
\begin{aligned}
\varliminf_{n \rightarrow \infty} \frac{\ln \sum_{|J|=n} d_{j}^{q}}{n \ln \rho} & =\varliminf_{n \rightarrow \infty} \frac{\ln \sum_{|J|=n} \mu\left(T_{J}(0, m)\right)^{q}}{\ln \left(m \rho^{n}\right)} \\
& \geq \varliminf_{h \rightarrow 0^{+}} \frac{\ln \sup \sum_{i} \mu\left(B_{h}\left(x_{i}\right)\right)^{q}}{\ln h}=\tau(q) .
\end{aligned}
$$

To prove the reverse inequality, we observe that, for $x_{i} \in \operatorname{supp}(\mu)$ and $h$ satisfying $m \rho^{n} \leq h<m \rho^{n-1}$, there exists some $J=\left(j_{1}, \ldots, j_{n}\right), j_{i}=0,1, \ldots, m-1$, such that

$$
B_{h}\left(x_{i}\right) \supseteq T_{J}[0, m) .
$$

This implies that, for each such $h$,

$$
\sup \sum_{i} \mu\left(B_{h}\left(x_{i}\right)\right)^{q} \leq \sum_{|J|=n} \mu\left(T_{J}(0, m)\right)^{q} .
$$

Consequently,

$$
\varliminf_{h \rightarrow 0^{+}} \frac{\ln \sup \sum_{i} \mu\left(B_{h}\left(x_{i}\right)\right)^{q}}{\ln h} \geq \varliminf_{n \rightarrow \infty} \frac{\ln \sum_{|J|=n} \mu\left(T_{J}[0, m)\right)^{q}}{\ln \left(m \rho^{n-1}\right)}=\underline{\lim _{n \rightarrow \infty}} \frac{\ln \sum_{|J|=n} d_{J}^{q}}{n \ln \rho}
$$

and the reverse inequality follows.

\section{DERIVATION OF THE FUNCTIONAL EQUATION}

Let

$$
S_{j} x=\rho x+b_{j}, \quad j=0,1, \ldots, m,
$$

where $0<\rho<1$ and $0=b_{0}<b_{1}<\cdots<b_{m}$. For any set of nonnegative probability weights $\left\{w_{j}\right\}_{j=0}^{m}$, we let $\mu$ be the corresponding self-similar measure defined as in (1.4). It is easy to see that $\operatorname{supp}(\mu) \subseteq[0, a]$, where $a=b_{m} /(1-\rho)$. Define

$$
T_{i} x=\rho^{n_{i}} x+d_{i}, \quad i=0,1, \ldots, \ell,
$$


where $n_{i}$ is a positive integer and $0=d_{0}<d_{1}<\cdots<d_{\ell}$. We assume that $\left\{T_{i}\right\}_{i=0}^{\ell}$ is a non-overlapping family, $\bigcup_{i=0}^{\ell} T_{i}[0, a] \supseteq \operatorname{supp}(\mu)$, and $\mu$ satisfies the following family of second-order identities: For $A \subseteq[0, a]$,

$$
\left[\begin{array}{c}
\mu\left(T_{0} T_{i} A\right) \\
\vdots \\
\mu\left(T_{\ell} T_{i} A\right)
\end{array}\right]=M_{i}\left[\begin{array}{c}
\mu\left(T_{0} A\right) \\
\vdots \\
\mu\left(T_{\ell} A\right)
\end{array}\right], \quad i=0,1, \ldots, \ell,
$$

where $M_{i}$ is an $(\ell+1) \times(\ell+1)$ constant matrix.

To calculate $\tau(q)$, we will use the following equivalent definition. For $q>0$,

$$
\tau(q)=\inf \left\{\alpha \mid \varlimsup_{h \rightarrow 0^{+}} \frac{1}{h^{1+\alpha}} \int_{-\infty}^{\infty} \mu\left(B_{h}(x)\right)^{q} d x>0\right\} .
$$

This can be derived by using [LN1, Proposition 3.1] (see also [La1], [LN2], [St]).

Our approach is to partition the collection $\left\{T_{i}\right\}_{i=0}^{\ell}$ into two subcollections, indexed by $\mathcal{I}_{0}$ and $\mathcal{I}_{1}$, so that if we let

$$
\Phi^{(\alpha)}(h)=\frac{1}{h^{1+\alpha}} \sum_{i \in I_{1}} \int_{0}^{a} \mu\left(B_{h}\left(T_{i} x\right)\right)^{q} d x,
$$

then $\Phi^{(\alpha)}(h)$ behaves like $1 / h^{1+\alpha} \int_{0}^{a} \mu\left(B_{h}(x)\right)^{q} d x$ as $h \rightarrow 0^{+}$and moreover, it satisfies a functional equation of the form

$$
\Phi^{(\alpha)}(h)=\sum_{k=0}^{\infty} c_{k}(q, \alpha) \Phi^{(\alpha)}\left(\frac{h}{\rho^{s_{k}}}\right)+o\left(h^{\varepsilon}\right),
$$

where $s_{k} \in \mathbb{N}$ and $\varepsilon>0$ (or more precisely (1.11)). The second-order identities (3.3) alone are not sufficient to fulfill these requirements; further restrictions need to be imposed.

We will assume that each of the subcollections $\left\{T_{i}\right\}_{i \in \mathcal{I}_{0}}$ and $\left\{T_{i}\right\}_{i \in \mathcal{I}_{1}}$ is equicontractive with

$$
n_{i}= \begin{cases}\tilde{n} & \text { if } i \in \mathcal{I}_{0} \\ n & \text { if } i \in \mathcal{I}_{1} .\end{cases}
$$

Moreover, we assume they satisfy the following basic conditions (C1), (C2), and (C3). (C1) governs the asymptotic behavior of $\Phi^{(\alpha)}(h)$. (C2) and (C3) are used in deriving (3.5). These conditions are satisfied by the interesting examples we consider, but not all of them are necessary. For simplicity we do not consider the more general framework, which will require a vector version of the renewal 
theorem. For $k \geq 1$, we denote by $\mathcal{I}_{0}^{k}$ the $k$-time Cartesian product $\mathcal{I}_{0} \times \cdots \times \mathcal{I}_{0}$; $\mathcal{I}_{1}^{k}$ is similarly defined.

(C1) For some $i \in \mathcal{I}_{1}, T_{i}[0, a]$ contains an interval of the form $S_{j_{1}} \cdots S_{j_{k}}[0, a]$ with $w_{j_{1}} \cdots w_{j_{k}}>0$. Furthermore, there exists a positive integer $t$ (chosen to be the smallest) and a subset $\mathbf{I} \subseteq\left\{\left(i_{1}, \ldots, i_{t}\right) \mid i_{j} \in \mathcal{I}_{1}\right\}$ such that, for $A \subseteq[0, a]$, conditions (C2) and (C3) below are satisfied:

(C2) Suppose for each $k=2, \ldots, t, \mathbf{I}_{k}^{0}$ denotes the collection of multi-indices $I=\left(i_{1}, \ldots, i_{k}\right)$ such that $i_{1} \in \mathcal{I}_{1}$ and $k$ is the smallest integer such that $I$ is not an initial segment of any member of $\mathbf{I}$. Then for each $I \in \mathbf{I}_{k}^{0}$ there exists an index $i=i(I) \in \mathcal{I}_{1}$, and a constant $c(I, i)$ depending only on $I$ and $i$ such that

$$
\mu\left(T_{I} A\right)=c(I, i) \mu\left(T_{i} A\right) .
$$

Moreover, for each $i \in \mathcal{I}_{1}$, the following sums are independent of $i$ :

$$
\begin{aligned}
& c_{k-1}^{0}(q):=\sum\left\{c(I, i)^{q} \mid I \in \mathbf{I}_{k}^{0}, i_{k} \in \mathcal{I}_{0}\right\}, \\
& c_{k-1}^{1}(q):=\sum\left\{c(I, i)^{q} \mid I \in \mathbf{I}_{k}^{0}, i_{k} \in \mathcal{I}_{1}\right\} .
\end{aligned}
$$

(C3) Suppose $I=\left(i_{1}, \ldots, i_{t}\right) \in \mathbf{I}$. Then for each $J=\left(j_{1}, \ldots, j_{k}\right) \in \mathcal{I}_{0}^{k}, k \geq 0$, and for each $j \in \mathcal{I}_{1}$, there exists an index $i=i(I, J, j) \in \mathcal{I}_{1}$ and a constant $c(I, J, i, j)$ depending only on $I, J, i$, and $j$ such that

$$
\mu\left(T_{I} T_{J} T_{j} A\right)=c(I, J, i, j) \mu\left(T_{i} A\right) .
$$

Moreover, for each $i \in \mathcal{I}_{1}$, the sum

$$
\widetilde{c}_{k}(q):=\sum\left\{c(I, J, i, j)^{q} \mid I \in \mathbf{I}, j \in \mathcal{I}_{1}, J \in \mathcal{I}_{0}^{k}\right\}
$$

is independent of $i$.

Remark 1. The second-order identities imply that $\mu\left(T_{I} A\right)$ and $\mu\left(T_{I} T_{J} T_{j} A\right)$ are linear combinations of $\mu\left(T_{i} A\right)$. (3.6) and (3.8) require in addition that they are multiples of some $\mu\left(T_{i} A\right)$. 
Remark 2. The assumptions corresponding to (3.7) and (3.9) can be dropped in a more general framework. Without these assumptions, a vector analogue of the functional equation (3.5) can still be set up and the vector renewal theorem of Lau et al. [LWC] can be used to obtain the desired results. However, for simplicity of exposition and for the purposes of this paper, we choose to include these assumptions and consider only the scalar case.

Remark 3. We can also consider the more general case which allows the $T_{i}$ to be non-equicontractive. Again, a vector version as mentioned in Remark 2 is required.

Example 1. The IFS defining the Bernoulli convolution associated with the golden ratio serves as a basic example for conditions (C1), (C2), and (C3): $S_{0}(x)=$ $\rho x, S_{1}(x)=\rho x+(1-\rho), \rho=(\sqrt{5}-1) / 2$. It is shown in [STZ] that the associated self-similar measure satisfies a family of second-order identities with respect to the following nonoverlapping maps

$$
T_{0} x=\rho^{2} x, \quad T_{1} x=\rho^{3} x+\rho^{2}, \quad T_{2} x=\rho^{2} x+\rho .
$$

(C1) holds if $w_{0}$ and $w_{1}$ are both positive. By taking $\mathcal{I}_{0}=\{0,2\}, \mathcal{I}_{1}=\{1\}$, and $\mathbf{I}=\{(1)\}$, it can be shown that (C2) and (C3) also hold. We refer the reader to [LN2] for details. Here we illustrate these conditions by another example.

Example 2. Let

$$
S_{j} x=\frac{1}{3} x+\frac{2}{3} j, \quad j=0,1,2,3
$$

and let $\mu$ be the self-similar measure defined by $\left\{S_{j}\right\}_{j=0}^{3}$ and positive weights $\left\{w_{j}\right\}_{j=0}^{3}$. Then $\operatorname{supp}(\mu)=[0,3]$. Define

$$
T_{i} x=\frac{1}{3} x+i, \quad i=0,1,2 .
$$

Then for any $A \subseteq[0,3]$,

$$
\left[\begin{array}{l}
\mu\left(T_{0} T_{i} A\right) \\
\mu\left(T_{1} T_{i} A\right) \\
\mu\left(T_{2} T_{i} A\right)
\end{array}\right]=M_{i}\left[\begin{array}{l}
\mu\left(T_{0} A\right) \\
\mu\left(T_{1} A\right) \\
\mu\left(T_{2} A\right)
\end{array}\right], \quad i=0,1,2,
$$


where $M_{0}, M_{1}$, and $M_{2}$ are given by (4.4) (see Proposition 4.2). Partition the index set $\{0,1,2\}$ for $T_{i}$ as $\mathcal{I}_{0}=\{0,2\}$ and $\mathcal{I}_{1}=\{1\}$. Then, since

$$
T_{1}[0,3]=[1,2] \supseteq S_{j_{1}} S_{j_{2}}[0,3]
$$

for, say, $\left(j_{1}, j_{2}\right)=(1,2)$ (or $\left.(1,3),(2,0),(2,1)\right),(\mathrm{C} 1)$ is satisfied.

To verify (C2) and (C3) we let $t=2$ and $\mathbf{I}=\{(1,1)\}$. For (C2) we need only consider $I=(1,0)$ or $(1,2)$. Let $A \subseteq[0,3]$. Then from the second-order identities, we have

$$
\mu\left(T_{1} T_{0} A\right)=w_{1} \mu\left(T_{1} A\right) \quad \text { and } \quad \mu\left(T_{1} T_{2} A\right)=w_{2} \mu\left(T_{1} A\right) .
$$

(See Proposition 4.3(a).) Hence (3.6) is satisfied with $k=2, i(I)=1$, and

$$
c(I, 1)= \begin{cases}w_{1} & \text { if } I=(1,0) \\ w_{2} & \text { if } I=(1,2) .\end{cases}
$$

(3.7) is also satisfied with $c_{1}^{0}(q)=c_{1}^{1}(q)=w_{1}^{q}+w_{2}^{q}$.

For (C3) we let $I=(1,1), J=\left(j_{1}, \ldots, j_{k}\right)$ with $j_{i}=0$ or 2 , and $j=1$. Then for any $A \subseteq[0,3]$, we will show that

$$
\mu\left(T_{I} T_{J} T_{1} A\right)=c_{J} \mu\left(T_{1} A\right)
$$

where

$$
c_{J}=\left[w_{2}, w_{1}\right] \widetilde{M}_{J}\left[\begin{array}{l}
w_{0} \\
w_{3}
\end{array}\right]
$$

and

$$
\widetilde{M}_{0}=\left[\begin{array}{ccc}
w_{0} & 0 & 0 \\
0 & 0 & 0 \\
w_{3} & 0 & w_{2}
\end{array}\right], \quad \widetilde{M}_{2}=\left[\begin{array}{ccc}
w_{1} & 0 & w_{0} \\
0 & 0 & 0 \\
0 & 0 & w_{3}
\end{array}\right] .
$$

(See Proposition 4.4.) Hence (3.8) holds with $c(I, J, i, j)=c_{J}$ and (3.9) is satisfied with $\widetilde{c}_{k}(q)=\sum_{|J|=k} c_{J}^{q}$.

Throughout the rest of this section we assume that (C1), (C2), and (C3) are satisfied. For $\alpha>0, h>0$, and $q>0$, define

$$
\varphi(h):=\sum_{i \in I_{1}} \int_{0}^{a} \mu\left(B_{h}\left(T_{i} x\right)\right)^{q} d x .
$$

Then

$$
\Phi^{(\alpha)}(h)=\frac{1}{h^{1+\alpha}} \varphi(h)
$$


Condition (C1) enables us to establish the asymptotic behavior of $\Phi^{(\alpha)}(h)$, so that if $0<\overline{\lim }_{h \rightarrow 0^{+}} \Phi^{(\alpha)}(h)<\infty$, then $\alpha=\tau(q)$.

Proposition 3.1. Assume that condition (C1) holds and let $q>0$. Then there exist some $k \in \mathbb{N}$ and constants $C_{1}, C_{2}>0$, independent of $h$, such that

$$
\frac{C_{1}}{\left(h / \rho^{k}\right)^{1+\alpha}} \int_{0}^{a} \mu\left(B_{h / \rho^{k}}(x)\right)^{q} d x \leqslant \Phi^{(\alpha)}(h) \leqslant \frac{C_{2}}{h^{1+\alpha}} \int_{0}^{a} \mu\left(B_{h}(x)\right)^{q} d x .
$$

Consequently, if $0<\overline{\lim }_{h \rightarrow 0^{+}} \Phi^{(\alpha)}(h)<\infty$, then $\alpha=\tau(q)$.

Proof. First, it is easy to see that

$$
\int_{0}^{a} \mu\left(B_{h}(x)\right)^{q} d x \geq \rho^{n} \sum_{i \in I_{1}} \int_{0}^{a} \mu\left(B_{h}\left(T_{i} x\right)\right)^{q} d x=\rho^{n} \varphi(h) .
$$

For the first inequality in (3.11), condition (C1) implies that there exists some $i \in \mathcal{I}_{1}$ such that $T_{i}[0, a] \supseteq S_{i_{1}} \cdots S_{i_{k}}[0, a]$. Hence,

$$
\begin{aligned}
\varphi(h) & \geq \int_{0}^{a} \mu\left(B_{h}\left(T_{i} x\right)\right)^{q} d x=\frac{1}{\rho^{n}} \int_{T_{i}[0, a]} \mu\left(B_{h}(x)\right)^{q} d x \\
& \geq \frac{1}{\rho^{n}} \int_{S_{i_{1}} \cdots S_{i_{k}}[0, a]} \mu\left(B_{h}(x)\right)^{q} d x .
\end{aligned}
$$

Iterating (1.4) yields

$$
\mu\left(B_{h}(x)\right)=\sum_{j_{1}, \ldots, j_{k}=0}^{m} w_{j_{1}} \cdots w_{j_{k}} \mu\left(B_{h / \rho^{k}}\left(S_{j_{k}}^{-1} \cdots S_{j_{1}}^{-1} x\right)\right) .
$$

Hence,

$$
\begin{aligned}
\varphi(h) & \geq \rho^{-n}\left(w_{i_{1}} \cdots w_{i_{k}}\right)^{q} \int_{S_{i_{1}} \cdots S_{i_{k}}[0, a]} \mu\left(B_{h / \rho^{k}}\left(S_{i_{k}}^{-1} \cdots S_{i_{1}}^{-1} x\right)\right)^{q} d x \\
& =\rho^{k-n}\left(w_{i_{1}} \cdots w_{i_{k}}\right)^{q} \int_{0}^{a} \mu\left(B_{h / \rho^{k}}(x)\right)^{q} d x .
\end{aligned}
$$

Combining this with (3.12) gives

$$
\begin{aligned}
& \frac{\rho^{-(k \alpha+n)}\left(w_{i_{1}} \cdots w_{i_{k}}\right)^{q}}{\left(h / \rho^{k}\right)^{1+\alpha}} \int_{0}^{a} \mu\left(B_{h / \rho^{k}}(x)\right)^{q} d x \\
& \quad \leq \Phi^{(\alpha)}(h) \leq \frac{\rho^{-n}}{h^{1+\alpha}} \int_{0}^{a} \mu\left(B_{h}(x)\right)^{q} d x .
\end{aligned}
$$


This establishes (3.11). As a consequence,

$$
0<\varlimsup_{h \rightarrow 0^{+}} \Phi^{(\alpha)}(h)<\infty \Leftrightarrow 0<\varlimsup_{h \rightarrow 0^{+}} \frac{1}{h^{1+\alpha}} \int_{0}^{a} \mu\left(B_{h}(x)\right)^{q} d x<\infty,
$$

and the last statement follows.

We now derive the functional equation (1.11). Let $\mathbf{I}$ and $\mathbf{I}_{k}^{0}(2 \leq k \leq t)$ be the index sets in conditions (C2) and (C3), and define $\mathbf{I}_{1}^{1}=\mathcal{I}_{1}$. For $2 \leq k \leq t$ we let $\mathbf{I}_{k}^{1}$ be the complement of $\mathbf{I}_{k}^{0}$ in $\mathcal{I}_{1}^{k-1} \times\left(\mathcal{I}_{0} \cup \mathcal{I}_{1}\right)$. Then for $h>0$,

$$
\begin{aligned}
\varphi(h)= & \sum_{i_{1} \in I_{1}} \int_{0}^{a} \mu\left(B_{h}\left(T_{i_{1}} x\right)\right)^{q} d x \\
= & \left(\sum_{\left(i_{1}, i_{2}\right) \in \mathbf{I}_{2}^{0}}+\sum_{\left(i_{1}, i_{2}\right) \in \mathbf{I}_{2}^{1}}\right) \rho^{n_{i_{2}}} \int_{0}^{a} \mu\left(B_{h}\left(T_{i_{1}} T_{i_{2}} x\right)\right)^{q} d x \\
= & \cdots \\
= & \sum_{\left(i_{1}, i_{2}\right) \in \mathbf{I}_{2}^{0}} \rho^{n_{i_{2}}} \int_{0}^{a} \mu\left(B_{h}\left(T_{i_{1}} T_{i_{2}} x\right)\right)^{q} d x+\cdots \\
& \quad+\sum_{\left(i_{1}, \ldots, i_{t}\right) \in \mathbf{I}_{t}^{0}} \rho^{(t-2) n+n_{i_{t}}} \int_{0}^{a} \mu\left(B_{h}\left(T_{i_{1}} \cdots T_{i_{t}} x\right)\right)^{q} d x \\
& \quad+\sum_{\left(i_{1}, \ldots, i_{t}\right) \in \mathbf{I}} \rho^{(t-1) n} \int_{0}^{a} \mu\left(B_{h}\left(T_{i_{1}} \cdots T_{i_{t}} x\right)\right)^{q} d x .
\end{aligned}
$$

Write $J=\left(j_{1}, \ldots, j_{k}\right)$ for $J \in \mathcal{I}_{0}^{k}, k \geq 0$, and let $N$ be any nonnegative integer. Then by iterating the last term of (3.13), we get

$$
\begin{aligned}
\varphi(h)= & \sum_{k=2}^{t} \sum_{I \in \mathbf{I}_{k}^{0}} \rho^{(k-2) n+n_{i_{k}}} \int_{0}^{a} \mu\left(B_{h}\left(T_{I} x\right)\right)^{q} d x \\
& +\sum_{k=0}^{N} \sum_{I \in \mathbf{I}} \sum_{|J|=k} \sum_{j \in \mathcal{I}_{1}} \rho^{t n+k \tilde{n}} \int_{0}^{a} \mu\left(B_{h}\left(T_{I} T_{J} T_{j} x\right)\right)^{q} d x+e^{1}(h) \\
= & (I)+(I I)+e^{1}(h),
\end{aligned}
$$

where the sum over $|J|=k$ runs over all $J \in \mathcal{I}_{0}^{k}$ and

$$
e^{1}(h)=\sum_{I \in \mathbf{I}} \sum_{|J|=N+1} \rho^{(t-1) n+(N+1) \tilde{n}} \int_{0}^{a} \mu\left(B_{h}\left(T_{I} T_{J} x\right)\right)^{q} d x .
$$


Note that for $2 \leq k \leq t$, if $B_{h / \rho^{(k-1) n+n_{i_{k}}}}(x) \subseteq[0, a]$, then condition (C2) ensures that for each $I=\left(i_{1}, \ldots, i_{k}\right) \in \mathbf{I}_{k}^{0}$, there exists an index $i=i(I) \in \mathcal{I}_{1}$ and a constant $c(I, i)$ such that

$$
\mu\left(B_{h}\left(T_{I} x\right)\right)=c(I, i) \mu\left(B_{h / \rho^{(k-2) n+n_{i_{k}}}}\left(T_{i} x\right)\right) .
$$

For $I \in \mathbf{I}_{k}^{0}$, let $s_{k}=s_{k}(I):=(k-1) n+n_{i_{k}}$. Then for $2 \leq k \leq t$,

$$
\begin{aligned}
\sum_{I \in \mathbf{I}_{k}^{0}} \rho^{s_{k}-n} \int_{0}^{a} \mu\left(B_{h}\left(T_{I} x\right)\right)^{q} d x \\
\quad=\sum_{I \in \mathbf{I}_{k}^{0}} \rho^{s_{k}-n}\left(\int_{0}^{h / \rho^{s_{k}}}+\int_{h / \rho^{s_{k}}}^{a-h / \rho^{s_{k}}}+\int_{a-h / \rho^{s_{k}}}^{a}\right) \mu\left(T_{I} B_{h / \rho^{s_{k}}}(x)\right)^{q} d x \\
\quad=\sum_{I \in \mathbf{I}_{k}^{0}} \rho^{s_{k}-n} C(I, i)^{q} \int_{0}^{a} \mu\left(B_{h / \rho^{s_{k}-n}}\left(T_{i(I)} x\right)\right)^{q} d x+e_{k}^{2}(h)-\tilde{e}_{k}^{2}(h),
\end{aligned}
$$

where

$$
\begin{aligned}
& e_{k}^{2}(h)=\sum_{I \in \mathbf{I}_{k}^{0}} \rho^{s_{k}-n}\left(\int_{0}^{h / \rho^{s_{k}}}+\int_{a-h / \rho^{s_{k}}}^{a}\right) \mu\left(B_{h}\left(T_{I} x\right)\right)^{q} d x \\
& \tilde{e}_{k}^{2}(h)=\sum_{I \in \mathbf{I}_{k}^{0}} \rho^{s_{k}-n} c(I, i)^{q}\left(\int_{0}^{h / \rho^{s_{k}}}+\int_{a-h / \rho^{s_{k}}}^{a}\right) \mu\left(B_{h / \rho^{s_{k}-n}}\left(T_{i(I)} x\right)\right)^{q} d x .
\end{aligned}
$$

By using the last statement in (C2), we can write the last expression in (3.15) as

$$
\begin{array}{r}
\rho^{(k-2) n+\tilde{n}} c_{k-1}^{0}(q) \varphi\left(\frac{h}{\rho^{(k-2) n+\tilde{n}}}\right)+\rho^{(k-1) n} c_{k-1}^{1}(q) \varphi\left(\frac{h}{\rho^{(k-1) n}}\right) \\
+e_{k}^{2}(h)-\tilde{e}_{k}^{2}(h) .
\end{array}
$$

Similarly, for $k \geq 0$ we let $\tilde{s}_{k}:=(t+1) n+k \tilde{n}$. If $B_{h / \rho^{s_{k}}}(x) \subseteq[0, a]$, then for $I \in \mathbf{I}, J=\left(j_{1}, \ldots, j_{k}\right) \in \mathcal{I}_{0}^{k}$, and $j \in \mathcal{I}_{1}$, we can apply (C3) to get 


$$
\begin{aligned}
& \int_{0}^{a} \mu\left(B_{h}\left(T_{I} T_{J} T_{j} x\right)\right)^{q} d x \\
& =\left(\int_{0}^{h / \rho^{\tilde{s}_{k}}}+\int_{h / \rho^{\tilde{s}_{k}}}^{a-h / \rho^{\tilde{s}_{k}}}+\int_{a-h / \rho^{\tilde{s}_{k}}}^{a}\right) \mu\left(T_{I} T_{J} T_{j} B_{h / \rho^{\tilde{s}_{k}}}(x)\right)^{q} d x \\
& =c(I, J, i, j)^{q} \int_{0}^{a} \mu\left(B_{h / \rho^{\tilde{s}_{k}-n}}\left(T_{i(I, J, j)} x\right)\right)^{q} d x \\
& +\left(\int_{0}^{h / \rho^{\tilde{s}_{k}}}+\int_{a-h / \rho^{\tilde{s}_{k}}}^{a}\right) \mu\left(B_{h}\left(T_{I} T_{J} T_{j} x\right)\right)^{q} d x \\
& -c(I, J, i, j)^{q}\left(\int_{0}^{h / \rho^{\tilde{s}_{k}}}+\int_{a-h / \rho^{\tilde{s}_{k}}}^{a}\right) \mu\left(B_{h / \rho^{\tilde{s}_{k}-n}}\left(T_{i(I, J, j)} x\right)\right)^{q} d x .
\end{aligned}
$$

Now suppose $N$ is the largest integer satisfying $0 \leq h \leq \rho^{\widetilde{s}_{N}}\left(=\rho^{(t+1) n+N \tilde{n}}\right)$. Then by the above derivation and the second statement in condition (C3), we have

$$
\begin{aligned}
(I I)=\sum_{k=0}^{N} \rho^{\tilde{s}_{k}-n} \widetilde{c}_{k}(q) \varphi\left(\frac{h}{\rho^{\tilde{s}_{k}}-n}\right) \\
\quad+\sum_{k=0}^{N} \sum_{I \in \mathbf{I}} \sum_{|J|=k} \sum_{j \in I_{1}} \rho^{\widetilde{s}_{k}-n}\left(\int_{0}^{h / \rho^{\tilde{s} k}}+\int_{a-h / \rho^{\tilde{s}_{k}}}^{a}\right) \mu\left(B_{h}\left(T_{I} T_{J} T_{j} x\right)\right)^{q} d x \\
\quad-\sum_{k=0}^{N} \rho^{\tilde{s}_{k}-n} \widetilde{c}_{k}(q) \sum_{j \in I_{1}}\left(\int_{0}^{h / \rho^{\tilde{s}_{k}}}+\int_{a-h / \rho^{\tilde{s}_{k}}}^{a}\right) \mu\left(B_{h / \rho^{\tilde{s}_{k}-n}}\left(T_{j} x\right)\right)^{q} d x
\end{aligned}
$$

Substituting this expression and (3.16) into (3.13) yields

$$
\text { (3.17) } \begin{aligned}
\varphi(h)=\sum_{k=2}^{t} \rho^{(k-2) n+\tilde{n}} c_{k-1}^{0}(q) \varphi\left(\frac{h}{\rho^{(k-2) n+\tilde{n}}}\right) \\
\\
+\sum_{k=2}^{t} \rho^{(k-1) n} c_{k-1}^{1}(q) \varphi\left(\frac{h}{\rho^{(k-1) n}}\right) \\
+\sum_{k=0}^{N} \rho^{t n+k \tilde{n}} \widetilde{c}_{k}(q) \varphi\left(\frac{h}{\rho^{t n+k \tilde{n}}}\right)+e^{1}(h)+e^{2}(h),
\end{aligned}
$$


where $e^{1}(h)$ is given in (3.14) and

$$
\begin{aligned}
e^{2}(h)=\sum_{k=2}^{t} & \left(e_{k}^{2}(h)-\widetilde{e}_{k}^{2}(h)\right) \\
& +\sum_{k=0}^{N} \sum_{I \in \mathbf{I}} \sum_{|J|=k} \sum_{j \in \mathcal{I}_{1}} \rho^{\widetilde{s}_{k}-n}\left(\int_{0}^{h / \rho^{\tilde{s}}}+\int_{a-h / \rho^{\tilde{s}_{k}}}^{a}\right) \mu\left(B_{h}\left(T_{I} T_{J} T_{j} x\right)\right)^{q} d x \\
& -\sum_{k=0}^{N} \rho^{\widetilde{s}_{k}-n} \widetilde{c}_{k}(q) \sum_{j \in \mathcal{I}_{1}}\left(\int_{0}^{h / \rho^{\tilde{s}_{k}}}+\int_{a-h / \rho^{\tilde{s_{k}}}}^{a}\right) \mu\left(B_{h / \rho^{\tilde{s}_{k}-n}}\left(T_{j} x\right)\right)^{q} d x .
\end{aligned}
$$

Recall that for $\alpha>0, \Phi^{(\alpha)}(h)=\left(1 / h^{1+\alpha}\right) \varphi(h)$. Hence (3.17) has the equivalent form (1.11) with $E(h)=\left(1 / h^{1+\alpha}\right)\left(e^{1}(h)+e^{2}(h)\right)$. This completes the derivation of (1.11).

Let $F(q, \alpha)$ and $D$ be defined as in (1.12). For $q>0$, let $\widetilde{\alpha}=\widetilde{\alpha}(q)$ be the unique $\alpha$ such that $(q, \alpha) \in \partial D$, the boundary of $D$.

Proposition 3.2. $D$ is convex. Consequently $\widetilde{\alpha}(q)$ is an increasing concave function of $q$.

Proof. For each $k$, the definition of $\widetilde{c}_{k}(q)$ allows us to write

$$
\tilde{c}_{k}(q)=\sum_{j=1}^{m_{k}} a(j, k)^{q}
$$

for some $0 \leq a(j, k) \leq 1$. The convexity of $D$ follows by applying Hölder's inequality. As $q$ increases, $\widetilde{c}_{k}(q)$ decreases and hence the corresponding $\widetilde{\alpha}(q)$ will increase.

\section{Proof of Theorem 1.1.}

The proof is similar to that of [LN2, Theorem 3.2]; we include it for completeness. We will apply the renewal theorem ([Fe], $[\mathrm{F}])$. Let $N$ be the largest integer satisfying $0<h \leq \rho^{(t+1) n+N \tilde{n}}$. Then for any $\alpha \geq 0$ and any $k \geq N+1$,

$$
\Phi^{(\alpha)}\left(\frac{h}{\rho^{t n+k \widetilde{n}}}\right) \leq\left(\frac{h}{\rho^{t n+k \widetilde{n}}}\right)^{-(1+\alpha)} \int_{0}^{a} \mu\left(B_{h / \rho^{t n+k \tilde{n}}}(x)\right)^{q} d x \leq a \rho^{-n(1+\alpha)} .
$$

Also, the hypotheses imply that $D$ is open, and hence for $(q, \alpha) \in D$ and $\varepsilon>0$ sufficiently small,

$$
\sum_{k=N+1}^{\infty} \rho^{-(t n+k \tilde{n}) \alpha} \widetilde{c}_{k}(q)=o\left(h^{\varepsilon}\right)
$$


These imply that

$$
E_{\infty}(h):=\sum_{k=N+1}^{\infty} \rho^{-(t n+k \tilde{n}) \alpha} \widetilde{c}_{k}(q) \Phi^{(\alpha)}\left(\frac{h}{\rho^{t n+k \tilde{n}}}\right)=o\left(h^{\varepsilon}\right) \quad \text { as } h \rightarrow 0^{+} .
$$

For $0<h \leq 1$, let $x=-\ln h \geq 0$, and define $f(x):=\Phi^{(\alpha)}\left(e^{-x}\right)$ if $x \geq 0$ and $f(x):=0$ if $x<0$. Then the formula for $\Phi^{(\alpha)}(h)$ becomes

$$
\begin{aligned}
f(x)=\sum_{k=1}^{t-1} \rho^{-((k-1) n+\tilde{n}) \alpha} c_{k}^{0}(q) f(x+((k-1) n+\tilde{n}) \ln \rho) & \\
& +\sum_{k=1}^{t-1} \rho^{-k n \alpha} c_{k}^{1}(q) f(x+k n \ln \rho) \\
& +\sum_{k=0}^{\infty} \rho^{-(t n+k \tilde{n}) \alpha} \tilde{c}_{k}(q) f(x+(t n+k \tilde{n}) \ln \rho) \\
& +E\left(e^{-x}\right)-E_{\infty}\left(e^{-x}\right) .
\end{aligned}
$$

Now assume that $\alpha$ satisfies $F(q, \alpha)=1$ and let $v$ be the measure with weight

$$
\begin{cases}\rho^{-((k-1) n+\tilde{n}) \alpha} c_{k}^{0}(q) & \text { at }-((k-1) n+\tilde{n}) \ln \rho, k=1, \ldots, t-1 \\ \rho^{-k n \alpha} c_{k}^{1}(q) & \text { at }-k n \ln \rho, k=1, \ldots, t-1 \\ \rho^{-(t n+k \tilde{n}) \alpha} \widetilde{c}_{k}(q) & \text { at }-(t n+k \tilde{n}) \ln \rho, k=0,1,2, \ldots\end{cases}
$$

Then $F(q, \alpha)=1$ implies that $v$ is a probability measure with support contained in $[0, \infty)$. Moreover, for $x>0,(3.18)$ can be written as

$f(x)=\int_{0}^{\infty} f(x-y) d v(y)+E\left(e^{-x}\right)-E_{\infty}\left(e^{-x}\right)=\int_{0}^{x} f(x-y) d v(y)+S(x)$,

where

$$
S(x)=E\left(e^{-x}\right)-E_{\infty}\left(e^{-x}\right)=o\left(e^{\varepsilon x}\right) \quad \text { as } x \rightarrow \infty .
$$

Since $D$ is open and $(q, \alpha) \in D$, the moment $\int_{0}^{\infty} y d v(y)$ is finite. The renewal theorem now implies that $0<\overline{\lim }_{h \rightarrow 0^{+}} \Phi^{(\alpha)}(h)<\infty$ (see [LN2, Theorem 3.2]). Proposition 3.1 implies that $\tau(q)=\alpha$. Lastly, note that $c_{k}^{0}(q), c_{k}^{1}(q), k=1, \ldots$, $t-1$ and $\tilde{c}_{k}(q), k=0,1,2, \ldots$ are linear combinations of exponential functions. Using again the fact that $(q, \alpha) \in D$ and $D$ is open, we conclude that $F$ is a $C^{1}$ function with $\partial F / \partial q<0$. The differentiability of $\tau$ now follows from the implicit function theorem. 
To simplify notation, for $k=0, \ldots, t-1$, we let

$$
c_{k}(q):= \begin{cases}c_{k}^{0}(q)+c_{k}^{1}(q) & \text { if } n \neq \tilde{n}, \\ c_{k}^{0}(q)\left(=c_{k}^{1}(q)\right) & \text { if } n=\tilde{n} .\end{cases}
$$

We can apply [N2, Theorem 1.1] to conclude that the dimension of $\mu$ is equal to $\tau^{\prime}(1)$. By implicitly differentiating $\tau(q)$ and using the fact that $\tau(1)=0$ we have

Corollary 3.3. Assume the same hypotheses of Theorem 1.1. Then

$$
\operatorname{dim}(\mu)=\tau^{\prime}(1)=\frac{\sum_{k=1}^{t-1} c_{k}^{\prime}(1)+\sum_{k=0}^{\infty} \widetilde{c}_{k}^{\prime}(1)}{\ln \rho\left(\sum_{k=1}^{t-1}\left(k n c_{k}(1)+(\tilde{n}-n) c_{k}^{0}(1)\right)+\sum_{k=0}^{\infty}(t n+k \tilde{n}) \tilde{c}_{k}(1)\right)} .
$$

\section{A SPECIAL FAMily OF IFS}

In this section we consider the family of IFS

$$
S_{j}(x)=\frac{1}{m} x+\frac{m-1}{m} j, \quad j=0,1, \ldots, m,
$$

where $m \geq 3$ is an "odd" integer. Let $\left\{w_{j}\right\}_{j=0}^{m}$ be a set of probability weights and let $\mu$ be the corresponding self-similar measure. We allow some $w_{j}$ to be zero so that we can consider the measure defined by a subfamily of $\left\{S_{j}\right\}_{j=0}^{m}$.

Define

$$
T_{i} x=\frac{1}{m} x+i, \quad i=0,1, \ldots, m-1 .
$$

Then by Proposition 2.1, $\mu$ satisfies a family of second-order identities with respect to $\left\{T_{i}\right\}_{i=0}^{m-1}$. In fact for $A \subseteq[0, m]$,

$$
\mu\left(T_{j} T_{i} A\right)=\sum_{\ell=0}^{m} w_{\ell} \mu\left(\frac{1}{m} A+i+m j-(m-1) \ell\right) .
$$

For $i=0,1, \ldots, m-1$, let $M_{i}$ be the matrix defined by (2.3) and let $m_{j, k}^{(i)}, j$, $k=0,1, \ldots, m-1$, denote the $(j+1, k+1)$-entry of $M_{i}$. Then, in view of (4.2),

$$
m_{j, k}^{(i)}= \begin{cases}w_{\ell} & \text { if } 0 \leq \ell \leq m \text { and } i+m j-(m-1) \ell=k, \\ 0 & \text { otherwise. }\end{cases}
$$


For example, for $m=3$,

(4.4) $\quad M_{0}=\left[\begin{array}{ccc}w_{0} & 0 & 0 \\ 0 & w_{1} & 0 \\ w_{3} & 0 & w_{2}\end{array}\right], \quad M_{1}=\left[\begin{array}{ccc}0 & w_{0} & 0 \\ w_{2} & 0 & w_{1} \\ 0 & w_{3} & 0\end{array}\right], \quad M_{2}=\left[\begin{array}{ccc}w_{1} & 0 & w_{0} \\ 0 & w_{2} & 0 \\ 0 & 0 & w_{3}\end{array}\right]$.

Let $\mathcal{I}_{0}=\{0, m-1\}$ and $\mathcal{I}_{1}=\{1, \ldots, m-2\}$. Then

$$
\varphi(h)=\sum_{i=1}^{m-2} \int_{0}^{m} \mu\left(B_{h}\left(T_{i} x\right)\right)^{q} d x .
$$

In order for (C1) to hold, we will assume that $w_{0}, w_{m}>0$ and that there exists $j_{0}$ $\left(1 \leq j_{0} \leq m-1\right)$ such that $w_{j_{0}}>0$. In fact, suppose either $w_{0}$ or $w_{m}$ vanishes. Then $\mu$ can be identified with the measure defined by $\left\{S_{0}, \ldots, S_{m-1}\right\}$ (in the case $\left.w_{m}=0\right)$. This family satisfies the OSC and the corresponding $\tau(q)$ can be computed easily by using (1.5). Similarly, if $w_{j}=0$ for all $1 \leq j \leq m-1$, then the reduced family $\left\{S_{0}, S_{m}\right\}$ will also satisfy the OSC.

Proposition 4.1. Assume $w_{0}, w_{m}>0$ and there exists $j_{0}\left(1 \leq j_{0} \leq m-1\right)$ such that $w_{j_{0}}>0$. Then condition (C1) is satisfied. Consequently the conclusion of Proposition 3.1 holds.

Proof. If $2 \leq j_{0} \leq m-1$, then $S_{j_{0}} S_{0}[0, m]$ is contained in $T_{j_{0}-1}[0, m]$. If $1 \leq j_{0} \leq m-2$, then $S_{j_{0}} S_{m}[0, m]$ is contained in $T_{j_{0}}[0, m]$.

The verification of condition (C2) is contained in the following two Propositions. For simplicity, we shall use the notation $i^{\prime}:=m-1-i$ throughout the rest of this paper.

Proposition 4.2. Let $A \subseteq[0, m]$. Then

(a) for $i=1, \ldots, m-2$,

$$
\mu\left(T_{i} T_{i^{\prime}} A\right)=w_{i+1} \mu\left(T_{0} A\right)+w_{i} \mu\left(T_{m-1} A\right) .
$$

(b)

(i)

$$
\left[\begin{array}{c}
\mu\left(T_{0} T_{0} A\right) \\
\mu\left(T_{0} T_{m-1} A\right) \\
\mu\left(T_{m-1} T_{0} A\right) \\
\mu\left(T_{m-1} T_{m-1} A\right)
\end{array}\right]=\left[\begin{array}{cc}
w_{0} & 0 \\
w_{1} & w_{0} \\
w_{m} & w_{m-1} \\
0 & w_{m}
\end{array}\right]\left[\begin{array}{c}
\mu\left(T_{0} A\right) \\
\mu\left(T_{m-1} A\right)
\end{array}\right]
$$


(ii) For $1 \leq k \leq m-2$,

$$
\mu\left(T_{0} T_{k} A\right)=w_{0} \mu\left(T_{k} A\right) \text { and } \mu\left(T_{m-1} T_{k} A\right)=w_{m} \mu\left(T_{k} A\right) .
$$

Proof. All identities follow by applying the definition of the $T_{i}$ 's and identities (1.4) and (4.2). We will prove (a) as an example:

$$
\mu\left(T_{i} T_{i^{\prime}} A\right)=\mu\left(\frac{1}{m^{2}} A+\frac{i^{\prime}}{m}+i\right)=\sum_{\ell=0}^{m} w_{\ell} \mu\left(\frac{1}{m} A+(i+1-\ell)(m-1)\right) .
$$

Since $(i+1-\ell)(m-1)$ is always an integral multiple of $m-1$, it belongs to the set $\{0,1, \ldots, m-1\}$ if and only if $\ell=i+1$ or $\ell=i$. Hence the sum reduces to $w_{i+1} \mu\left(T_{0} A\right)+w_{i} \mu\left(T_{m-1} A\right)$.

Proposition 4.3. Let $A \subseteq[0, m]$. Then

(a) for $i=1, \ldots, m-2$,

$$
\mu\left(T_{i} T_{j} A\right)= \begin{cases}w_{i} \mu\left(T_{j+i} A\right) & \text { if } 0 \leq j<i^{\prime} \\ w_{i+1} \mu\left(T_{j-i^{\prime}} A\right) & \text { if } i^{\prime}<j \leq m-1 .\end{cases}
$$

(b)

$$
\sum_{i=1}^{m-2} \sum_{\substack{j=0 \\ j \neq i^{\prime}}}^{m-1} \mu\left(T_{i} T_{j} A\right)^{q}=\left(\sum_{j=1}^{m-1} w_{j}^{q}\right) \sum_{i=1}^{m-2} \mu\left(T_{i} A\right)^{q} .
$$

Proof.

(a) By (4.2),

$$
\mu\left(T_{i} T_{j} A\right)=\sum_{\ell=0}^{m} w_{\ell} \mu\left(\frac{1}{m} A+j+m i-(m-1) \ell\right) .
$$

Let $s=s(i, j, \ell):=j+m i-(m-1) \ell$. If $0 \leq j<i^{\prime}(=m-1-i)$, then $m i-(m-1) \ell \leq s<(m-1)(1+i-\ell)$, and $s$ belongs to $\{0,1, \ldots, m-1\}$ if and only if $\ell=i$. Hence $s=j+i$ and $\mu\left(T_{i} T_{j} A\right)=w_{i} \mu\left(T_{j+i} A\right)$. On the other hand, if $i^{\prime}<j \leq m-1$, then $(m-1)(1+i-\ell)<s \leq$ $m i+(m-1)(1-\ell) ; s$ belongs to $\{0,1, \ldots, m-1\}$ if and only if $\ell=i+1$ and the result follows. 
(b) Applying part (a) to the left-hand side of (4.6), we see that it is equal to

$$
\begin{array}{r}
\sum_{i=1}^{m-2}\left(\sum_{j=0}^{m-i-2} w_{i}^{q} \mu\left(T_{j+i} A\right)^{q}+\sum_{j=m-i}^{m-1} w_{i+1}^{q} \mu\left(T_{j-i^{\prime}} A\right)^{q}\right) \\
=\sum_{i=1}^{m-2}\left(\sum_{j=i}^{m-2} w_{i}^{q} \mu\left(T_{j} A\right)^{q}+\sum_{j=1}^{i} w_{i+1}^{q} \mu\left(T_{j} A\right)^{q}\right) \\
=\sum_{i=1}^{m-2}\left(\sum_{j=1}^{m-2} w(i, j)^{q} \mu\left(T_{j} A\right)^{q}+w_{i+1}^{q} \mu\left(T_{i} A\right)^{q}\right),
\end{array}
$$

where

$$
w(i, j):= \begin{cases}w_{i+1} & \text { if } j<i \\ w_{i} & \text { if } j \geq i\end{cases}
$$

After re-grouping, it equals

$$
\sum_{j=1}^{m-2}\left(\sum_{i=1}^{m-2} w(i, j)^{q}+w_{j+1}^{q}\right) \mu\left(T_{j} A\right)^{q}=\sum_{j=1}^{m-2}\left(\sum_{i=1}^{m-1} w_{i}^{q}\right) \mu\left(T_{j} A\right)^{q} .
$$

Remark. If $A \subseteq[-1, m]$ then the conclusion of Proposition 4.3(a) still holds except when $(i, j)=(1,0)$ (in the case $\left.0 \leq j<i^{\prime}\right)$ or when $j+i=m$ (in the case $\left.i^{\prime}<j \leq m-1\right)$. In these cases we have

$$
\mu\left(T_{i} T_{j} A\right)= \begin{cases}w_{i-1} \mu\left(\frac{1}{m} A+m\right)+w_{i} \mu\left(T_{j+i} A\right) & \text { if }(i, j)=(1,0), \\ w_{i} \mu\left(\frac{1}{m} A+m\right)+w_{i+1} \mu\left(T_{j-i^{\prime}} A\right) & \text { if } j+i=m .\end{cases}
$$

The analogous result for $A \subseteq[0, m+1]$ can also be established.

Let $t=2, n=\tilde{n}=1, \mathbf{I}=\left\{\left(i, i^{\prime}\right): i=1, \ldots, m-2\right\}$, and $c_{1}(q)=c_{1}^{0}(q)=$ $c_{1}^{1}(q)=\sum_{j=1}^{m-1} w_{j}^{q}$. Proposition 4.3 shows that condition (C2) is satisfied.

In order to verify condition (C3), we will first introduce the following auxiliary matrices defined in terms of the $M_{i}$ 's. For $i=0$ or $m-1$, let $\widetilde{M}_{i}$ be the matrix formed from $M_{i}$ by keeping its first and last rows and assigning 0 to all other entries. For $1 \leq i \leq m-2$, let $\widetilde{M}_{i}$ denote the matrix formed from $M_{i}$ by 
keeping its $(m-i)$-th row and assigning 0 to all other entries. For example, when $m=3$,

$$
\widetilde{M}_{0}=\left[\begin{array}{ccc}
w_{0} & 0 & 0 \\
0 & 0 & 0 \\
w_{3} & 0 & w_{2}
\end{array}\right], \quad \widetilde{M}_{1}=\left[\begin{array}{ccc}
0 & 0 & 0 \\
w_{2} & 0 & w_{1} \\
0 & 0 & 0
\end{array}\right], \quad \widetilde{M}_{2}=\left[\begin{array}{ccc}
w_{1} & 0 & w_{0} \\
0 & 0 & 0 \\
0 & 0 & w_{3}
\end{array}\right] .
$$

For $J=\left(j_{1}, \ldots, j_{k}\right), k \geq 0$, and $j_{i}=0$ or $m-1$, we define

$$
c_{i, J}=\left[w_{i+1}, \mathbf{0}, w_{i}\right] \widetilde{M}_{J}\left[\begin{array}{c}
w_{0} \\
\mathbf{0} \\
w_{m}
\end{array}\right] \quad\left(=\mathbf{e}_{i} M_{i^{\prime}} \widetilde{M}_{J}\left[\begin{array}{c}
w_{0} \\
\mathbf{0} \\
w_{m}
\end{array}\right]\right),
$$

for $i=1, \ldots, m-2$, where $\mathbf{0}=(0, \ldots, 0)$ denotes the zero vector in $\mathbb{R}^{m-2}$, and we recall that $\mathbf{e}_{i}$ denotes the unit vector in $\mathbb{R}^{m}$ whose $(i+1)$-st coordinate is 1 .

Proposition 4.4. For $A \subseteq[0, m]$, the following higher-order relations hold:

(a) For $i=0$ or $m-1$,

$$
\left[\begin{array}{c}
\mu\left(T_{0} T_{i} A\right) \\
\mathbf{0} \\
\mu\left(T_{m-1} T_{i} A\right)
\end{array}\right]=\widetilde{M}_{i}\left[\begin{array}{c}
\mu\left(T_{0} A\right) \\
\mathbf{0} \\
\mu\left(T_{m-1} A\right)
\end{array}\right] .
$$

(b) For $i=1, \ldots, m-2$,

$$
\mu\left(T_{i} T_{i^{\prime}} A\right)=\mathbf{e}_{i} \widetilde{M}_{i^{\prime}}\left[\begin{array}{c}
\mu\left(T_{0} A\right) \\
\mathbf{0} \\
\mu\left(T_{m-1} A\right)
\end{array}\right]
$$

(c) For $J=\left(j_{1}, \ldots, j_{k}\right), k \geq 0$, and $j_{i}=0$ or $m-1$,

(i) $\mu\left(T_{i} T_{i^{\prime}} T_{J} T_{j} A\right)=c_{i, J} \mu\left(T_{j} A\right)$, for $j=1, \ldots, m-2$;

(ii) $\mu\left(T_{i} T_{i^{\prime}} T_{J} A\right) \leq C \cdot c_{i, J}$, for the constant

$$
C=\max \left\{\frac{1}{1-w_{1}}, \frac{1}{1-w_{m-1}}\right\} .
$$


Proof.

(a) Proposition 4.2(b)(i) shows that, for $i=0$ or $m-1, \mu\left(T_{0} T_{i} A\right)$ and $\mu\left(T_{m-1} T_{i} A\right)$ can be expressed as linear combinations of $\mu\left(T_{0} A\right)$ and $\mu\left(T_{m-1} A\right)$. This together with the special form of $M_{0}$ and $M_{m-1}$ implies that

$$
\left[\begin{array}{c}
\mu\left(T_{0} T_{i} A\right) \\
\mathbf{0} \\
\mu\left(T_{m-1} T_{i} A\right)
\end{array}\right]=M_{i}\left[\begin{array}{c}
\mu\left(T_{0} A\right) \\
\mathbf{0} \\
\mu\left(T_{m-1} A\right)
\end{array}\right]=\widetilde{M}_{i}\left[\begin{array}{c}
\mu\left(T_{0} A\right) \\
\mathbf{0} \\
\mu\left(T_{m-1} A\right)
\end{array}\right] .
$$

(b) For $i=1, \ldots, m-2$, Proposition $4.2(\mathrm{a})$ shows that $\mu\left(T_{i} T_{i^{\prime}} A\right)$ is a linear combination of $\mu\left(T_{0} A\right)$ and $\mu\left(T_{m-1} A\right)$. Hence

$$
\mu\left(T_{i} T_{i^{\prime}} A\right)=\mathbf{e}_{i} M_{i^{\prime}}\left[\begin{array}{c}
\mu\left(T_{0} A\right) \\
\mathbf{0} \\
\mu\left(T_{m-1} A\right)
\end{array}\right]=\mathbf{e}_{i} \widetilde{M}_{i^{\prime}}\left[\begin{array}{c}
\mu\left(T_{0} A\right) \\
\mathbf{0} \\
\mu\left(T_{m-1} A\right)
\end{array}\right],
$$

and the result follows.

(c)(i) Let $J=\left(j_{1}, \ldots, j_{k}\right), k \geq 0, j_{i}=0$ or $m-1$. Then for $j=1, \ldots$, $m-2$, by applying part (b) and repeatedly applying part (a), we have

$$
\begin{aligned}
\mu\left(T_{i} T_{i^{\prime}} T_{J} T_{j} A\right) & =\mathbf{e}_{i} \widetilde{M}_{i^{\prime}} \widetilde{M}_{J}\left[\begin{array}{c}
\mu\left(T_{0} T_{j} A\right) \\
\mathbf{0} \\
\mu\left(T_{m-1} T_{j} A\right)
\end{array}\right]=\mathbf{e}_{i} \widetilde{M}_{i^{\prime}} \widetilde{M}_{J}\left[\begin{array}{c}
w_{0} \\
\mathbf{0} \\
w_{m}
\end{array}\right] \mu\left(T_{j} A\right) \\
& =c_{i, J} \mu\left(T_{j} A\right) .
\end{aligned}
$$

(The second equality follows from Proposition 4.2(b)(ii).)

(c)(ii) By using the same argument as in the proof of (i), we get

$$
\mu\left(T_{i} T_{i^{\prime}} T_{J} A\right)=\mathbf{e}_{i} \widetilde{M}_{i^{\prime}} \widetilde{M}_{J}\left[\begin{array}{c}
\mu\left(T_{0} A\right) \\
\mathbf{0} \\
\mu\left(T_{m-1} A\right)
\end{array}\right] \leq \mathbf{e}_{i} \widetilde{M}_{i^{\prime}} \widetilde{M}_{J}\left[\begin{array}{c}
\mu[0,1] \\
\mathbf{0} \\
\mu[m-1, m]
\end{array}\right] .
$$

Applying (1.4) yields $\mu[0,1]=w_{0} \mu[0, m]+w_{1} \mu[0,1]$, and hence

$$
\mu[0,1]=\frac{w_{0}}{1-w_{1}} .
$$


Similarly,

$$
\mu[m-1, m]=\frac{w_{m}}{1-w_{m-1}} .
$$

The result follows by combining (4.8), (4.9) and (4.10).

Proposition 4.4(c)(i) shows that (C3) holds with $\tilde{c}_{k}(q)=\sum_{i=1}^{m-2} \sum_{|J|=k} c_{i, J}^{q}$. As a result, we can apply (1.11) to obtain the following functional equation:

$$
\begin{aligned}
\Phi^{(\alpha)}(h)=m^{\alpha} & \left(\sum_{i=1}^{m-1} w_{i}^{q}\right) \Phi^{(\alpha)}(m h) \\
& +\sum_{k=0}^{N} m^{(k+2) \alpha} \sum_{i=1}^{m-2}\left(\sum_{|J|=k} c_{i, J}^{q}\right) \Phi^{(\alpha)}\left(m^{k+2} h\right)+E(h),
\end{aligned}
$$

where $N$ is the largest integer satisfying $h<1 /\left(2 m^{N+3}\right)$, and $E(h)$ is some error term (see Section 3 and the Appendix).

We will now use a change of basis to replace the $m \times m$ matrices $\widetilde{M}_{0}$ and $\widetilde{M}_{m-1}$ in the definition of $c_{i, J}$ by the following $2 \times 2$ matrices:

$$
P_{0}=\left[\begin{array}{cc}
w_{0} & 0 \\
w_{m} & w_{m-1}
\end{array}\right], \quad P_{m-1}=\left[\begin{array}{cc}
w_{1} & w_{0} \\
0 & w_{m}
\end{array}\right] .
$$

Proposition 4.5. Let $k \geq 0$ and $J=\left(j_{1}, \ldots, j_{k}\right)$ where $j_{i}=0$ or $m-1$. Then for $i=1, \ldots, m-2$,

$$
c_{i, J}=\left[w_{i+1}, w_{i}\right] P_{J}\left[\begin{array}{c}
w_{0} \\
w_{m}
\end{array}\right]
$$

Proof. Let $S$ be the $m \times m$ matrix obtained by interchanging the second and the last rows of the $m \times m$ identity matrix. Then $S^{-1}=S$. If we define $Q_{0}=S^{-1} \widetilde{M}_{0} S$ and $Q_{m-1}=S^{-1} \widetilde{M}_{m-1} S$, then and a direct calculation yields

$$
Q_{0}=\left[\begin{array}{ccccc}
w_{0} & 0 & 0 & \cdots & 0 \\
w_{m} & w_{m-1} & 0 & \cdots & 0 \\
0 & 0 & 0 & \cdots & 0 \\
\vdots & \vdots & \vdots & \ddots & \vdots \\
0 & 0 & 0 & \cdots & 0
\end{array}\right], \quad Q_{m-1}=\left[\begin{array}{ccccc}
w_{1} & w_{0} & 0 & \cdots & 0 \\
0 & w_{m} & 0 & \cdots & 0 \\
0 & 0 & 0 & \cdots & 0 \\
\vdots & \vdots & \vdots & \ddots & \vdots \\
0 & 0 & 0 & \cdots & 0
\end{array}\right] .
$$


Hence

$$
\begin{aligned}
c_{i, J} & =\left[w_{i+1}, \mathbf{0}, w_{i}\right]\left(S Q_{j_{1}} S^{-1}\right) \cdots\left(S Q_{j_{k}} S^{-1}\right)\left[\begin{array}{c}
w_{0} \\
\mathbf{0} \\
w_{m}
\end{array}\right] \\
& =\left[w_{i+1}, \mathbf{0}, w_{i}\right] S\left[\begin{array}{cc}
P_{J} & \mathbf{0} \\
\mathbf{0} & \mathbf{0}
\end{array}\right] S^{-1}\left[\begin{array}{c}
w_{0} \\
\mathbf{0} \\
w_{m}
\end{array}\right]=\left[w_{i+1}, w_{i}\right] P_{J}\left[\begin{array}{c}
w_{0} \\
w_{m}
\end{array}\right] .
\end{aligned}
$$

Let

$$
F(q, \alpha)=m^{\alpha} \sum_{i=1}^{m-1} w_{i}^{q}+\sum_{k=0}^{\infty} m^{(k+2) \alpha}\left(\sum_{i=1}^{m-2} \sum_{|J|=k} c_{i, J}^{q}\right),
$$

and

$$
D=\{(q, \alpha) \mid q>0 \text { and } F(q, \alpha)<\infty\}
$$

Then we have the following key lemma. Since it involves quite a lot of technical estimations, we will postpone its proof to the Appendix.

Lemma 4.6. Fixing $q>0$, if $(q, \widetilde{\alpha}) \in \partial D$, then $F(q, \alpha)$ tends to $\infty$ as $\alpha$ increases to $\tilde{\alpha}$. Moreover, for the unique $\alpha$ satisfying $F(q, \alpha)=1$, there exists $\varepsilon>0$ such that $E(h)=o\left(h^{\varepsilon}\right)$ as $h \rightarrow 0^{+}$.

Theorem 1.2 now follows by combining Theorem 3.3, Lemma 4.6, and the derivations in this section. Furthermore, applying Theorem 1.2 and Corollary 3.3 with $t=2, n=\tilde{n}=1, c_{1}(q)=\sum_{j=1}^{m-1} w_{j}^{q}$ and $\widetilde{c}_{k}(q)=\sum_{i=1}^{m-2} \sum_{|J|=k} c_{i, J}^{q}$, we obtain the following result concerning the dimension of $\mu$. (We use the convention $x \ln x:=0$ in the case $x=0$.)

Corollary 4.7. Under the same hypotheses of Theorem 1.2,

$$
\operatorname{dim}(\mu)=\tau^{\prime}(1)=\frac{-\sum_{i=1}^{m-1} w_{i} \ln w_{i}-\sum_{k=0}^{\infty} \sum_{i=1}^{m-2} \sum_{|J|=k} c_{i, J} \ln c_{i, J}}{\ln m\left(\sum_{i=1}^{m-1} w_{i}+\sum_{k=0}^{\infty}(k+2) \sum_{i=1}^{m-2} \sum_{|J|=k} c_{i, J}\right)} .
$$




\section{RESULTS FOR INTEGER $q$}

For $q$ equal to a positive integer, we can reduce the expression for $F(q, \alpha)$ in Section 4 to a polynomial. This greatly simplifies the computations of $\tau(q)$ for small positive integers $q$. Let

$$
\begin{aligned}
& a_{i j}= \begin{cases}\left(\begin{array}{l}
i \\
j
\end{array}\right) w_{0}^{q-i} w_{m-1}^{j} w_{m}^{i-j} & \text { if } 0 \leq j \leq i \leq q, \\
0 & \text { if } 0 \leq i<j \leq q,\end{cases} \\
& b_{i j}= \begin{cases}\left(\begin{array}{l}
q-i \\
j-i
\end{array}\right) w_{0}^{j-i} w_{1}^{q-j} w_{m}^{i} & \text { if } 0 \leq i \leq j \leq q, \\
0 & \text { if } 0 \leq j<i \leq q,\end{cases}
\end{aligned}
$$

and define $A_{q}=\left(a_{i j}\right)_{0 \leq i, j \leq q}+\left(b_{i j}\right)_{0 \leq i, j \leq q}$. For example,

$$
\begin{aligned}
A_{1} & =\left[\begin{array}{cc}
w_{0} & 0 \\
w_{m} & w_{m-1}
\end{array}\right]+\left[\begin{array}{cc}
w_{1} & w_{0} \\
0 & w_{m}
\end{array}\right] \\
A_{2} & =\left[\begin{array}{ccc}
w_{0}^{2} & 0 & 0 \\
w_{0} w_{m} & w_{0} w_{m-1} & 0 \\
w_{m}^{2} & 2 w_{m-1} w_{m} & w_{m-1}^{2}
\end{array}\right]+\left[\begin{array}{ccc}
w_{1}^{2} & 2 w_{0} w_{1} & w_{0}^{2} \\
0 & w_{1} w_{m} & w_{0} w_{m} \\
0 & 0 & w_{m}^{2}
\end{array}\right] .
\end{aligned}
$$

Theorem 5.1. For $q \in \mathbb{N}$, the equation $F(q, \alpha)=1$ in Theorem 1.2 can be reduced to

$$
\begin{array}{r}
z \sum_{i=1}^{m-1} w_{i}^{q}+z^{2} \sum_{i=1}^{m-2}\left[\left(\begin{array}{l}
q \\
0
\end{array}\right) w_{i+1}^{q}\left(\begin{array}{l}
q \\
1
\end{array}\right) w_{i+1}^{q-1} w_{i} \cdots\left(\begin{array}{l}
q \\
q
\end{array}\right) w_{i}^{q}\right] \\
\times\left(I-z A_{q}\right)^{-1}\left[\begin{array}{c}
w_{0}^{q} \\
w_{0}^{q-1} w_{m} \\
\vdots \\
w_{m}^{q}
\end{array}\right]=1,
\end{array}
$$

with $z=m^{\alpha}$. Furthermore, $\tau(q)=\ln z / \ln m$, where $z$ is the smallest real root of equation (5.1). 
Proof. Rewrite $F(q, \alpha)$ as

$$
F(q, \alpha)=m^{\alpha} \sum_{i=1}^{m-1} w_{i}^{q}+\sum_{i=1}^{m-2}\left(\sum_{k=0}^{\infty} m^{(k+2) \alpha} \sum_{|J|=k} c_{i, J}^{q}\right) .
$$

Let $e_{1}=[1,0], e_{2}=[0,1]$ and for $i=1, \ldots, m-2$, let

$$
s_{i, k}=\sum_{|J|=k} c_{i, J}^{q}
$$

For $q>0$ by applying Proposition 4.5 and the binomial theorem, we have

$$
\begin{aligned}
s_{i, k} & =\sum_{|J|=k}\left(\left(w_{i+1} e_{1}+w_{i} e_{2}\right) P_{J}\left[\begin{array}{c}
w_{0} \\
w_{m}
\end{array}\right]\right)^{q} \\
& =\sum_{|J|=k} \sum_{j=0}^{q}\left(\begin{array}{l}
q \\
j
\end{array}\right)\left(w_{i+1} e_{1} P_{J}\left[\begin{array}{c}
w_{0} \\
w_{m}
\end{array}\right]\right)^{q-j}\left(w_{i} e_{2} P_{J}\left[\begin{array}{c}
w_{0} \\
w_{m}
\end{array}\right]\right)^{j} \\
& =\sum_{j=0}^{q}\left(\begin{array}{l}
q \\
j
\end{array}\right) w_{i+1}^{q-j} w_{i}^{j} \gamma_{j}^{(k)},
\end{aligned}
$$

where

$$
\gamma_{j}^{(k)}:=\sum_{|J|=k}\left(e_{1} P_{J}\left[\begin{array}{c}
w_{0} \\
w_{m}
\end{array}\right]\right)^{q-j}\left(e_{2} P_{J}\left[\begin{array}{c}
w_{0} \\
w_{m}
\end{array}\right]\right)^{j}, \quad j=0,1, \ldots, q .
$$

By using the identities

$$
\begin{array}{ll}
e_{1} P_{0}=w_{0} e_{1}, & e_{1} P_{m-1}=w_{1} e_{1}+w_{0} e_{2}, \\
e_{2} P_{0}=w_{m} e_{1}+w_{m-1} e_{2}, & e_{2} P_{m-1}=w_{m} e_{2},
\end{array}
$$

and the binomial theorem again, we get the following recursion formula for $\gamma_{j}^{(k)}$ (see also [LN2, Theorem 4.1]):

$$
\gamma_{j}^{(k+1)}=\sum_{\ell=0}^{j} a_{j \ell} \gamma_{\ell}^{(k)}+\sum_{\ell=j}^{q} b_{j \ell} \gamma_{\ell}^{(k)}, \quad j=0,1, \ldots, q .
$$


In matrix form

$$
\left[\begin{array}{c}
\gamma_{0}^{(k+1)} \\
\gamma_{1}^{(k+1)} \\
\vdots \\
\gamma_{q}^{(k+1)}
\end{array}\right]=A_{q}\left[\begin{array}{c}
\gamma_{0}^{(k)} \\
\gamma_{1}^{(k)} \\
\vdots \\
\gamma_{q}^{(k)}
\end{array}\right]
$$

By repeatedly applying this recursion formula, we get

$$
s_{i, k}=\left[\left(\begin{array}{l}
q \\
0
\end{array}\right) w_{i+1}^{q}\left(\begin{array}{l}
q \\
1
\end{array}\right) w_{i+1}^{q-1} w_{i} \cdots\left(\begin{array}{l}
q \\
q
\end{array}\right) w_{i}^{q}\right] A_{q}^{k}\left[\begin{array}{c}
w_{0}^{q} \\
w_{0}^{q-1} w_{m} \\
\vdots \\
w_{m}^{q}
\end{array}\right] .
$$

The result follows by substituting this expression into (5.2).

\section{Convolution of the Cantor measure}

Let $\mu$ denote the 3 -fold convolution of the usual Cantor measure, which is defined by the similitudes

$$
S_{j} x=\frac{1}{3} x+\frac{2}{3} j, \quad j=0,1,2,3,
$$

with probability weights $\frac{1}{8}, \frac{3}{8}, \frac{3}{8}$, and $\frac{1}{8}$ respectively. By Theorem 1.2, for $q>0$, $\tau(q)$ is defined by the equation

$$
2 \cdot 3^{\alpha}\left(\frac{3}{8}\right)^{q}+\sum_{k=0}^{\infty} 3^{(k+2) \alpha}\left(\sum_{|J|=k} c_{J}^{q}\right)=1,
$$

where $J=\left(j_{1}, \ldots, j_{k}\right), j_{i}=0$ or 2 ,

$$
c_{J}=\frac{3}{64}[1,1] P_{J}\left[\begin{array}{l}
1 \\
1
\end{array}\right], \quad P_{0}=\frac{1}{8}\left[\begin{array}{ll}
1 & 0 \\
1 & 3
\end{array}\right], \quad \text { and } \quad P_{2}=\frac{1}{8}\left[\begin{array}{ll}
3 & 1 \\
0 & 1
\end{array}\right] .
$$

Moreover, $\tau$ is differentiable on $(0, \infty)$. By Corollary 4.7 , the dimension of $\mu$ is equal to

$$
\tau^{\prime}(1)=-\frac{\frac{3}{4} \ln \frac{3}{8}+\sum_{k=0}^{\infty} \sum_{|J|=k} c_{J} \ln c_{J}}{\ln 3\left(\frac{3}{4}+\sum_{k=0}^{\infty}(k+2) \sum_{|J|=k} c_{J}\right)} .
$$


To simplify the sum in the denominator we define

$$
g(\alpha):=\sum_{k=0}^{\infty} 3^{(k+2) \alpha}\left(\sum_{|J|=k} c_{J}\right) .
$$

By a direct calculation,

$$
g(\alpha)=\frac{3^{2 \alpha+1}}{64}[1,1] \sum_{k=0}^{\infty}\left(3^{\alpha}\left(P_{0}+P_{1}\right)\right)^{k}\left[\begin{array}{l}
1 \\
1
\end{array}\right]=\frac{8 \cdot 3^{2 \alpha+1}-3^{3 \alpha+2}}{4\left(64-64 \cdot 3^{\alpha}+5 \cdot 3^{2 \alpha+1}\right)} .
$$

By calculating the derivative of $g(\alpha)$ at $\alpha=0$, we get $\sum_{k=0}^{\infty}(k+2) \sum_{|J|=k} c_{J}=\frac{11}{12}$. Hence $\operatorname{dim}(\mu)$ is simplified to

$$
\operatorname{dim}(\mu)=\frac{9 \ln \frac{3}{8}+12 \sum_{k=0}^{\infty} \sum_{|J|=k} c_{J} \ln c_{J}}{-20 \ln 3} .
$$

The computation for $k=30$ in the above formula yields $\operatorname{dim}(\mu) \approx 0.9884 \ldots$.

For $q$ equal to a positive integer, we apply Theorem 5.1 to obtain the following results for $\tau(q)$ and the $L^{q}$-dimension (where $z=3^{\alpha}$ ). They coincide with those obtained in [FLN] by using a different method.

$q F(q, \alpha)=1$

$245 z^{2}-832 z+2048=0$

$3387 z^{2}-18944 z+131072=0$

$426973 z^{3}-16883712 z^{2}+1853882368 z-34359738368=0$

$5818667 z^{3}-1185546240 z^{2}+347355480064 z-17592186044416=0$

$$
\tau(q) \quad L^{q}-\operatorname{dim}
$$

0.9766281250 .976628125

1.9306492240 .965324612

2.8654620010 .955154000

3.7857738090 .946443452

For the rest of this section, we calculate the lower $L^{\infty}$-dimension of $\mu$. Let

$$
\widetilde{P}_{0}=\left[\begin{array}{ll}
1 & 0 \\
1 & 3
\end{array}\right] \text { and } \quad \widetilde{P}_{2}=\left[\begin{array}{ll}
3 & 1 \\
0 & 1
\end{array}\right] \text {. }
$$

Proposition 6.1. Let $k \geq 1$ and $J=\left(j_{1}, \ldots, j_{k}\right), j_{i}=0$ or 2. Then:

(a) $\widetilde{P}_{0}^{k}=\left[\begin{array}{cc}1 & 0 \\ \left(3^{k}-1\right) / 2 & 3^{k}\end{array}\right]$ and $\widetilde{P}_{2}^{k}=\left[\begin{array}{cc}3^{k} & \left(3^{k}-1\right) / 2 \\ 0 & 1\end{array}\right]$.

(b) $J=(2, \ldots, 2)$ and $J=(0, \ldots, 0)$ maximize, respectively, the first and the second column sums over all $\widetilde{P}_{J}$. 
(c) $[1,1] \widetilde{P}_{J}\left[\begin{array}{l}1 \\ 1\end{array}\right]$ is maximum when $J=(0, \ldots, 0)$ or $J=(2, \ldots, 2)$. Consequently,

$$
\max \left\{c_{J}:|J|=k\right\}=\frac{3^{k+2}+3}{2 \cdot 8^{k+2}}<\left(\frac{3}{8}\right)^{k+2}
$$

(d) $\sum_{k=0}^{\infty} \sum_{|J|=k} c_{J}=\frac{1}{4}$.

Proof. Both (a) and (b) follow directly by induction on $k$. To prove the first statement of (c) we again apply induction on $k$. The statement obviously holds when $k=1$. Assume that it holds for $|J|=k$. We want to maximize

$$
[1,1] \widetilde{P}_{J} \widetilde{P}_{j}\left[\begin{array}{l}
1 \\
1
\end{array}\right], \quad j=0,2
$$

for all possible $J$. In the case $j=0$,

$$
[1,1] \widetilde{P}_{J} \widetilde{P}_{0}\left[\begin{array}{l}
1 \\
1
\end{array}\right]=[1,1] \widetilde{P}_{J}\left[\begin{array}{l}
1 \\
1
\end{array}\right]+3[1,1] \widetilde{P}_{J}\left[\begin{array}{l}
0 \\
1
\end{array}\right]
$$

By induction hypothesis the first term is maximized when $J=(0, \ldots, 0)$ or $J=$ $(2, \ldots, 2)$. The second term is 3 times the second column sum of $\widetilde{P}_{J}$ and by part (b), it is maximized by $J=(0, \ldots, 0)$. Hence the quantity in (6.3) with $j=0$ is maximized by $J=(0, \ldots, 0)$. The case for $j=2$ is similar. The second statement of (c) follows from the first statement and part (a). (d) can be proved directly as follows:

$$
\begin{aligned}
\sum_{k=0}^{\infty} \sum_{|J|=k} c_{J} & =\frac{3}{64} \sum_{k=0}^{\infty}[1,1]\left(P_{0}+P_{2}\right)^{k}\left[\begin{array}{l}
1 \\
1
\end{array}\right] \\
& =\frac{3}{64}[1,1]\left(I-\left(P_{0}+P_{2}\right)\right)^{-1}\left[\begin{array}{l}
1 \\
1
\end{array}\right] \\
& =\frac{3}{64}[1,1]\left(\frac{8}{15}\left[\begin{array}{ll}
4 & 1 \\
1 & 4
\end{array}\right]\right)\left[\begin{array}{l}
1 \\
1
\end{array}\right]=\frac{1}{4}
\end{aligned}
$$

Alternatively it can be obtained by putting $q=1$ and $\alpha=\tau(1)=0$ into (6.1). 
Theorem 6.2. $\underline{\operatorname{dim}}_{\infty}(\mu)=\frac{\ln (8 / 3)}{\ln 3}(\approx 0.89278926 \ldots)$.

Proof. It suffices to show that

$$
\lim _{q \rightarrow \infty} \frac{\tau(q)}{q}=\frac{\ln (8 / 3)}{\ln 3} .
$$

(See [LN1, Proposition 3.4].) We first observe that for all $q>0,(6.1)$ implies that

$$
2 \cdot 3^{\tau(q)}\left(\frac{3}{8}\right)^{q}<1
$$

Taking logarithms on both sides and then letting $q \rightarrow \infty$, we have

$$
\lim _{q \rightarrow \infty} \frac{\tau(q)}{q} \leq \frac{\ln (8 / 3)}{\ln 3} .
$$

To prove the reverse inequality, we let $\ell=\lim _{q \rightarrow \infty}[\tau(q)] / q$ and suppose

$$
\ell<\frac{\ln (8 / 3)}{\ln 3} \text {. }
$$

We claim that the first term in the series (6.1) with $\alpha=\tau(q)$ would tend to 0 as $q \rightarrow \infty$. In fact we notice that

$$
3^{\tau(q)}\left(\frac{3}{8}\right)^{q}=\left(3^{\tau(q) / q} \cdot \frac{3}{8}\right)^{q}
$$

and by assumption (6.5),

$$
\lim _{q \rightarrow \infty} 3^{T(q) / q}\left(\frac{3}{8}\right)=3^{\ell}\left(\frac{3}{8}\right)<1 .
$$

Consequently, $2 \cdot 3^{\tau(q)}\left(\frac{3}{8}\right)^{q} \rightarrow 0$ as $q \rightarrow \infty$.

The claim above implies that there exists $q_{o}>0$ such that, for all $q \geq q_{o}$,

$$
\begin{aligned}
\frac{1}{2} & \leq \sum_{k=0}^{\infty} 3^{(k+2) \tau(q)}\left(\sum_{|J|=k} c_{J}^{q}\right) \\
& \leq \sum_{k=0}^{\infty} 3^{(k+2) \tau(q)}\left(\max \left\{c_{J}:|J|=k\right\}\right)^{q-1}\left(\sum_{|J|=k} c_{J}\right) \\
& \leq \sum_{k=0}^{\infty} 3^{(k+2) \tau(q)}\left(\frac{3}{8}\right)^{(k+2)(q-1)}\left(\sum_{|J|=k} c_{J}\right) \\
& \leq\left[\sup _{k \geq 0}\left(3^{\tau(q)}\left(\frac{3}{8}\right)^{q-1}\right)^{k+2}\right] \cdot \frac{1}{4} .
\end{aligned}
$$


This forces $3^{\tau(q)}\left(\frac{3}{8}\right)^{q-1} \geq 1$ for all $q \geq q_{o}$, which yields

$$
\lim _{q \rightarrow \infty} \frac{\tau(q)}{q} \geq \frac{\ln (8 / 3)}{\ln 3} .
$$

This contradicts (6.5) and thus the reverse inequality is also established.

\section{A.ppendix. Proof of Lemma 4.6}

Some techniques in this section have been used in [LN2]. For the sake of completeness, we include the proofs and indicate the main differences. We first show that $D$ is open and that for each $q>0, F(q, \alpha)$ can take arbitrarily large values.

Proposition A.1. For each fixed $q>0$, if $(q, \widetilde{\alpha}) \in \partial D$, then $F(q, \alpha)$ tends to $\infty$ as $\alpha$ increases to $\widetilde{\alpha}$. In particular, $D$ is open.

Proof. For each $i=1, \ldots, m-2$, we define

$F_{i}(q, \alpha)=\sum_{k=0}^{\infty} m^{(k+2) \alpha}\left(\sum_{|J|=k} c_{i, J}^{q}\right) \quad$ and $\quad D_{i}=\left\{(q, \alpha) \mid q>0, F_{i}(q, \alpha)<\infty\right\}$

Then

$$
F(q, \alpha)=m^{\alpha} \sum_{i=1}^{m-1} w_{i}^{q}+\sum_{i=1}^{m-2} F_{i}(q, \alpha)
$$

We first prove that, if $w_{i}, w_{i+1}>0$, then the analogous conclusions of the proposition hold for the pair $\left(F_{i}, D_{i}\right)$. For such an $i$, let $d_{i}=\min \left\{w_{i}, w_{i+1}\right\}$ and $d_{0}=\min \left\{w_{0}, w_{m}\right\}$. Then

$$
F_{i}(q, \alpha)=d_{0}^{q} d_{i}^{q} \sum_{k=0}^{\infty} m^{(k+2) \alpha} \sum_{|J|=k}\left(\left[\widetilde{w}_{i+1}, \widetilde{w}_{i}\right] P_{J}\left[\begin{array}{c}
\widetilde{w}_{0} \\
\widetilde{w}_{m}
\end{array}\right]\right)^{q},
$$

where $\widetilde{w}_{i}=w_{i} / d_{i}$ and $\widetilde{w}_{i+1}, \widetilde{w}_{0}, \widetilde{w}_{m}$ are similarly defined. Note that $\widetilde{w}_{i}, \widetilde{w}_{i+1}$, $\widetilde{w}_{0}, \widetilde{w}_{m} \geq 1$. For $k \geq 0$, let

$$
s_{k}:=\sum_{|J|=k}\left(\left[\widetilde{w}_{i+1}, \widetilde{w}_{i}\right] P_{J}\left[\begin{array}{c}
\widetilde{w}_{0} \\
\widetilde{w}_{m}
\end{array}\right]\right)^{q} .
$$

It can be checked directly that $s_{\ell+k} \leq s_{\ell} s_{k}$ for any integers $\ell, k \geq 0$. We can now apply the same proof as in [LN2, Proposition 2.5] to conclude that $D_{i}$ is open and that $F_{i}(q, \alpha)$ tends to $\infty$ as $\alpha$ tends to $\partial D_{i}$. 
Next, we claim that if $w_{j}, w_{j+1}>0$ and $D_{i}$ is as above, then $D_{j}=D_{i}$. In fact, $\partial D_{j}$ is determined uniquely by the number

$$
R_{j}:=\varlimsup_{k \rightarrow \infty}\left(\sum_{|J|=k} c_{j, J}^{q}\right)^{1 / k} .
$$

Let $d_{j}=\min \left\{w_{j}, w_{j+1}\right\}$. Then $d_{j}>0$ and the inequality

$$
\begin{gathered}
\varlimsup_{k \rightarrow \infty}\left(d_{j}^{q} \sum_{|J|=k}\left(\left[\widetilde{w}_{j+1}, \widetilde{w}_{j}\right] P_{J}\left[\begin{array}{c}
w_{0} \\
w_{m}
\end{array}\right]\right)^{q}\right)^{1 / k} \\
\geq \varlimsup_{k \rightarrow \infty}\left(\sum_{|J|=k}\left(\left[w_{i+1}, w_{i}\right] P_{J}\left[\begin{array}{c}
w_{0} \\
w_{m}
\end{array}\right]\right)^{q}\right)^{1 / k}
\end{gathered}
$$

yields $R_{j} \geq R_{i}$. By symmetry we have $R_{j}=R_{i}$ and hence $\partial D_{j}=\partial D_{i}$.

Lastly we show that, even if one of $w_{j}$ or $w_{j+1}$ is zero (but not both), we still have $D_{j}=D_{i}$. It suffices to show that

$$
\begin{aligned}
\varlimsup_{k \rightarrow \infty}\left(\sum_{|J|=k}\left([1,1] P_{J}\left[\begin{array}{c}
w_{0} \\
w_{m}
\end{array}\right]\right)^{q}\right)^{1 / k} & =\varlimsup_{k \rightarrow \infty}\left(\sum_{|J|=k}\left([1,0] P_{J}\left[\begin{array}{c}
w_{0} \\
w_{m}
\end{array}\right]\right)^{q}\right)^{1 / k} \\
& =\varlimsup_{k \rightarrow \infty}\left(\sum_{|J|=k}\left([0,1] P_{J}\left[\begin{array}{c}
w_{0} \\
w_{m}
\end{array}\right]\right)^{q}\right)^{1 / k} .
\end{aligned}
$$

The second equality can be easily established. To see the first equality we notice that

$$
\begin{aligned}
& \sum_{|J|=k}\left([1,1] P_{J}\left[\begin{array}{c}
w_{0} \\
w_{m}
\end{array}\right]\right)^{q} \\
& \quad \leq \gamma_{q} \sum_{|J|=k}\left(\left([1,0] P_{J}\left[\begin{array}{c}
\widetilde{w}_{0} \\
\widetilde{w}_{m}
\end{array}\right]\right)^{q}+\left([0,1] P_{J}\left[\begin{array}{c}
\widetilde{w}_{0} \\
\widetilde{w}_{m}
\end{array}\right]\right)^{q}\right)
\end{aligned}
$$

where $\gamma_{q}=\max \left\{1,2^{q-1}\right\}$. We now recall the fact that if $\left\{a_{k}\right\},\left\{b_{k}\right\}$ are two sequences of nonnegative numbers, then

$$
\varlimsup_{k \rightarrow \infty}\left(a_{k}+b_{k}\right)^{1 / k}=\max \left\{\varlimsup_{k \rightarrow \infty}\left(a_{k}\right)^{1 / k}, \varlimsup_{k \rightarrow \infty}\left(b_{k}\right)^{1 / k}\right\} .
$$


Thus by taking the $k$-th root on both sides and then letting $k$ tend to $\infty$, we get

$$
\varlimsup_{k \rightarrow \infty}\left(\sum_{|J|=k}\left([1,1] P_{J}\left[\begin{array}{c}
w_{0} \\
w_{m}
\end{array}\right]\right)^{q}\right)^{1 / k} \leq \varlimsup_{k \rightarrow \infty}\left(\sum_{|J|=k}\left([1,0] P_{J}\left[\begin{array}{c}
w_{0} \\
w_{m}
\end{array}\right]\right)^{q}\right)^{1 / k} .
$$

The reverse inequality is obvious. Consequently, $F_{i}(q, \alpha)$ still tends to $\infty$ as $\alpha$ tends to $\partial D_{i}$. We conclude that if $w_{i+1} \neq 0$ or $w_{i} \neq 0$, then $D_{i}=D$ and the proof is complete.

Recall that for $i=1, \ldots, m-2$, we let $i^{\prime}=m-1-i$.

Proposition A.2. Let $J=\left(j_{1}, \ldots, j_{k}\right)$ where $k \geq 0, j_{\ell}=0$, or $m-1$, and let $1 \leq i, j \leq m-2$. Then

(a) There exists a constant $C>0$ such that, for $B \subseteq[-1,0]$ or $B \subseteq[m, m+1]$,

$$
c_{i, J} \mu\left(T_{j} B\right) \leq \mu\left(T_{i} T_{i^{\prime}} T_{J} T_{j} B\right) \leq C \cdot c_{i, J} \mu\left(T_{j} B\right) .
$$

(C can be taken to be $\max \left\{1+w_{m-1} / w_{0}, 1+w_{1} / w_{m}\right\}$.)

(b) For $B \subseteq[-1,0]$, the following hold:

(i) $\mu\left(T_{i} T_{i^{\prime}} T_{J} T_{m-1} B\right) \leq C \cdot c_{i, J}$, where we can take $C=\max \left\{1 /\left(1-w_{1}\right)\right.$, $\left.1 /\left(1-w_{m-1}\right)\right\}$

(ii) if $J=\left(J^{\prime}, m-1,0, \ldots, 0\right),\left|J^{\prime}\right|=\ell$, then $\mu\left(T_{i} T_{i^{\prime}} T_{J} T_{0} B\right) \leq w_{m-1}^{k-\ell+1} c_{i, J^{\prime}}$;

(iii) if $J=(0, \ldots, 0)$, then $\mu\left(T_{i} T_{i^{\prime}} T_{J} T_{0} B\right) \leq w_{1} w_{m-1}^{k+2}$.

For $B \subseteq[m, m+1]$, similar statements hold by interchanging the roles of $T_{0}$ and $T_{m-1}$.

Proof.

(a) For any $B \subseteq[-1, m+1]$ and any $j=1, \ldots, m-2$, we have $T_{j} B \subseteq[0, m]$. Hence, as in the proof of Proposition 4.4(c)(ii),

$$
\mu\left(T_{i} T_{i^{\prime}} T_{J} T_{j} B\right)=\left[w_{i+1}, \mathbf{0}, w_{i}\right] \widetilde{M}_{J}\left[\begin{array}{c}
\mu\left(T_{0} T_{j} B\right) \\
\mathbf{0} \\
\mu\left(T_{m-1} T_{j} B\right)
\end{array}\right] .
$$

For $B \subseteq[-1,0]$, a direct calculation yields

$$
\begin{aligned}
\mu\left(T_{0} T_{j} B\right) & =w_{0} \mu\left(T_{j} B\right), \\
\mu\left(T_{m-1} T_{j} B\right) & =w_{m} \mu\left(T_{j} B\right)+w_{m-1} \mu\left(T_{j} B+m-1\right) .
\end{aligned}
$$


The first inequality in the proposition follows immediately. For the second inequality we observe that

$$
\mu\left(T_{j} B+m-1\right)= \begin{cases}w_{m} \mu(B+m) & \text { if } j=1 \\ 0 & \text { if } j>1,\end{cases}
$$

while $\mu\left(T_{j} B\right)=w_{0} \mu(B+m)+w_{1} \mu(B+1)$ if $j=1$. Hence $\mu\left(T_{j} B+m-1\right) \leq$ $\left(w_{m} / w_{0}\right) \mu\left(T_{j} B\right)$ and consequently

$$
\mu\left(T_{m-1} T_{j} B\right) \leq w_{m}\left(1+\frac{w_{m-1}}{w_{0}}\right) \mu\left(T_{j} B\right) .
$$

Substituting this and the first equality in (A.2) into (A.1), we get the desired result with $C=1+w_{m-1} / w_{0}$. The proof for $B \subseteq[m, m+1]$ is similar and yields $C=1+w_{1} / w_{m}$.

(b)(i) Since $T_{m-1} B \subseteq[0, m]$, the inequality follows from Proposition 4.4(c) (ii).

(b)(ii) Let $J=\left(J^{\prime}, m-1,0, \ldots, 0\right)$ with $\left|J^{\prime}\right|=\ell$. Then $T_{m-1} T_{0}^{k-\ell} B \subseteq$ $[0, m]$ and hence

$$
\mu\left(T_{i} T_{i^{\prime}} T_{J} T_{0} B\right)=\left[w_{i+1}, \mathbf{0}, w_{i}\right] \widetilde{M}_{J^{\prime}}\left[\begin{array}{c}
\mu\left(T_{0} T_{m-1} T_{0}^{k-\ell} B\right) \\
\mathbf{0} \\
\mu\left(T_{m-1} T_{m-1} T_{0}^{k-\ell} B\right)
\end{array}\right] .
$$

The desired inequality follows by putting the following identities into (A.3).

$$
\begin{aligned}
\mu\left(T_{0} T_{m-1} T_{0}^{k-\ell} B\right) & =w_{0} w_{m-1}^{k-\ell+1} \mu(B+m-1), \\
\mu\left(T_{m-1} T_{m-1} T_{0}^{k-\ell} B\right) & =w_{m} w_{m-1}^{k-\ell+1} \mu(B+m-1) .
\end{aligned}
$$

(c) For $J=(0, \ldots, 0)$, by a similar calculation as above, we have

$$
\mu\left(T_{i} T_{i^{\prime}} T_{J} T_{0} B\right)=w_{i} w_{m-1}^{k+2} \mu(B+m-1) \leq w_{i} w_{m-1}^{k+2} .
$$

Define

$$
a_{1}=\left\{\begin{array}{ll}
\left|\frac{\ln w_{1}}{\ln m}\right| & \text { if } w_{1} \geq w_{m} \\
\left|\frac{\ln w_{m}}{\ln m}\right| & \text { if } w_{1}<w_{m}
\end{array}, \quad a_{m-1}=\left\{\begin{array}{ll}
\left|\frac{\ln w_{m-1}}{\ln m}\right| & \text { if } w_{m-1} \geq w_{0} \\
\left|\frac{\ln w_{0}}{\ln m}\right| & \text { if } w_{m-1}<w_{0}
\end{array},\right.\right.
$$


and for $i=0, m$, or $2 \leq i \leq m-2$, define

$$
a_{i}=\left|\frac{\ln w_{i}}{\ln m}\right|, \quad \text { if } w_{i}>0 .
$$

If $w_{i}=0$, we use the convention $a_{i}=\infty$.

Proposition A.3. There exist constants $C_{1}, C_{2}>0$ so that, for all $0<h<1$, the following statements hold:

(a) For $i=0, m$, or $2 \leq i \leq m-2$,

$$
C_{1} h^{a_{i}} \leq \mu\left(B_{h}(i)\right) \leq C_{2} h^{a_{i}}
$$

(b) For $i=1$ or $m-1$, let $i_{0}=m$ if $i=1$ and let $i_{0}=0$ if $i=m-1$. Then

(i) $C_{1} h^{a_{i}} \leq \mu\left(B_{h}(i)\right) \leq C_{2} h^{a_{i}}$ if $w_{i} \neq w_{i_{0}}$, and

(ii) $C_{1}|\ln h| h^{a_{i}} \leq \mu\left(B_{h}(i)\right) \leq C_{2}|\ln h| h^{a_{i}}$, if $w_{i}=w_{i_{0}}$.

Proof.

(a) Let $k$ be the integer satisfying $1 / m^{k+1}<h \leq 1 / m^{k}$. For the given values of $i$, a direct calculation using (1.4) yields

$$
\mu\left(B_{h}(i)\right)=w_{i}^{k} \mu\left(B_{m^{k} h}(i)\right) \geq w_{i}^{k+2} \mu\left(B_{m^{k+2} h}(i)\right) .
$$

$B_{m^{k+2} h}(i)$ contains the interval $[0, m]$ and therefore $w_{i}^{k+2} \leq \mu\left(B_{h}(i)\right) \leq w_{i}^{k}=$ $m^{-k a_{i}}$. The result follows.

(b) We need only consider the case $i=1$; the case $i=m-1$ is similar. Let $k$ be the integer such that $1 / m^{k+1}<h \leq 1 / m^{k}$. Then by using $\mu\left(B_{m^{\ell+1} h}(m)\right)=$ $w_{m}^{k-\ell-1} \mu\left(B_{m^{k} h}(m)\right)$ for $0 \leq \ell \leq k-1$, we have

$$
\begin{aligned}
\mu\left(B_{h}(1)\right) & =w_{1}^{k} \mu\left(B_{m^{k} h}(1)\right)+w_{0} \sum_{\ell=0}^{k-1} w_{1}^{\ell} \mu\left(B_{m^{\ell+1} h}(m)\right) \\
& =w_{1}^{k} \mu\left(B_{m^{k} h}(1)\right)+w_{0}\left(\sum_{\ell=0}^{k-1} w_{m}^{k-\ell-1} w_{1}^{\ell}\right) \mu\left(B_{m^{k} h}(m)\right) \\
& \geq w_{1}^{k+2} \mu\left(B_{m^{k+2} h}(1)\right)+w_{0} w_{m}^{2}\left(\sum_{\ell=0}^{k-1} w_{m}^{k-\ell-1} w_{1}^{\ell}\right) \mu\left(B_{m^{k+2} h}(m)\right) .
\end{aligned}
$$


Since both $B_{m^{k+2} h}(1)$ and $B_{m^{k+2} h}(m)$ contain the interval $[0, m]$, the last inequality gives the following lower bound estimations:

$$
\mu\left(B_{h}(1)\right) \geq \begin{cases}w_{1}^{k+2}+w_{0} w_{m}^{2} w_{1}^{k-1} \sum_{\ell=0}^{k-1}\left(\frac{w_{m}}{w_{1}}\right)^{\ell} & \text { if } w_{1}>w_{m}, \\ w_{1}^{k+2}+w_{0} k w_{1}^{k+1} & \text { if } w_{1}=w_{m}, \\ w_{1}^{k+2}+w_{0} w_{m}^{k+1} \sum_{\ell=0}^{k-1}\left(\frac{w_{1}}{w_{m}}\right)^{\ell} & \text { if } w_{1}<w_{m} .\end{cases}
$$

By using $\mu\left(B_{h}(1)\right) \leq w_{1}^{k}+w_{0} \sum_{\ell=0}^{k-1} w_{m}^{k-\ell-1} w_{1}^{\ell}$, similar upper bound estimations can be obtained. This proves part (b).

Proposition A.4. Let $q>0$. If $w_{i}>0$ and $\alpha \geq q a_{i}$ for some $i=0,1, \ldots$, $m$, then $F(q, \alpha)>1$.

Proof. For $i=2, \ldots, m-2, \alpha \geq q a_{i}$ if and only if $m^{\alpha} \geq w_{i}^{-q}$. Hence $F(q, \alpha)>w_{i}^{q} m^{\alpha} \geq 1$. For $i=1$ and $w_{1} \geq w_{m}$, the same result holds. Hence we assume that $w_{1}<w_{m}$. Note that if $J=(m-1, \ldots, m-1)$ with $|J|=k$, then $c_{1, J} \geq w_{1} w_{m}^{k+1}$. In fact, for such $J$,

$$
\begin{aligned}
c_{1, J} & =\left[w_{2}, w_{1}\right]\left[\begin{array}{cc}
w_{1} & w_{0} \\
0 & w_{m}
\end{array}\right]^{k}\left[\begin{array}{c}
w_{0} \\
w_{m}
\end{array}\right] \\
& =\left[w_{2}, w_{1}\right]\left[\begin{array}{cc}
w_{1}^{k} w_{0} \sum_{i=0}^{k-1} w_{1}^{k-1-i} w_{m}^{i} \\
0 & w_{m}^{k}
\end{array}\right]\left[\begin{array}{c}
w_{0} \\
w_{m}
\end{array}\right] \\
& \geq w_{1} w_{m}^{k+1} .
\end{aligned}
$$

Now let $q>0$ and $\alpha \geq q a_{1}$. Then $m^{\alpha} w_{m}^{q} \geq 1$ and

$$
F(q, \alpha) \geq \sum_{k=0}^{\infty} m^{(k+2) \alpha}\left(\sum_{|J|=k} c_{1, J}^{q}\right) \geq \sum_{k=0}^{\infty} m^{(k+2) \alpha} w_{1}^{q} w_{m}^{(k+1) q}=\infty .
$$

The case for $i=m-1$ is similar. The cases for $i=0$ or 2 can also be proved by modifying the above argument slightly. 
Now we can prove the required error estimations for $e^{1}(h)$ and $e^{2}(h)$. We first rewrite $e^{2}(h)$ as

$$
\begin{aligned}
e^{2}(h)= & \frac{1}{m} \sum_{i=1}^{m-2} \sum_{\substack{j=0 \\
j \neq i^{\prime}}}^{m-1}\left(\int_{0}^{m^{2} h}+\int_{m-m^{2} h}^{m}\right) \mu\left(B_{h}\left(T_{i} T_{j} x\right)\right)^{q} d x \\
& +\sum_{k=0}^{N} \sum_{|J|=k} \sum_{i, j=1}^{m-2} \frac{1}{m^{k+2}}\left(\int_{0}^{m^{k+3} h}+\int_{m-m^{k+3} h}^{m}\right) \mu\left(B_{h}\left(T_{i} T_{i^{\prime}} T_{J} T_{j} x\right)\right)^{q} d x \\
& -\frac{1}{m} \sum_{i=1}^{m-2}\left(\sum_{j=1}^{m-1} w_{j}^{q}\right)\left(\int_{0}^{m^{2} h}+\int_{m-m^{2} h}^{m}\right) \mu\left(B_{m h}\left(T_{i} x\right)\right)^{q} d x \\
& -\sum_{k=0}^{N} \sum_{|J|=k} \sum_{i, j=1}^{m-2} \frac{c_{i, J}^{q}}{m^{k+2}}\left(\int_{0}^{m^{k+3} h}+\int_{m-m^{k+3} h}^{m}\right) \mu\left(B_{m^{k+2} h}\left(T_{j} x\right)\right)^{q} d x \\
= & (I)+(I I)-(I I I)-(I V) .
\end{aligned}
$$

Lemma A.5. Suppose $q>0$ and $(q, \alpha) \in D$ such that $\alpha<q a_{j}$ for all $j=0$, $1, \ldots, m$. For $0<h<1$ let $N$ be the largest integer satisfying $h<1 /\left(2 m^{N+3}\right)$. Then there exists $\varepsilon>0$ such that $e^{2}(h)=o\left(h^{1+\alpha+\varepsilon}\right)$ as $h \rightarrow 0^{+}$.

Proof. Choose $\varepsilon>0$ so that $\alpha+\varepsilon<q a_{j}$ for all $j=0,1, \ldots, m$ and that $(q, \alpha+\varepsilon) \in D$. Let us first estimate (II). By symmetry, we need only estimate a generic term

$$
\sum_{k=0}^{N} \sum_{|J|=k} \frac{1}{m^{k+2}} \int_{0}^{m^{k+3} h} \mu\left(B_{h}\left(T_{i} T_{i^{\prime}} T_{J} T_{j} x\right)\right)^{q} d x .
$$

For $0 \leq x \leq m^{k+3} h, B_{m^{k+3} h}(x) \subseteq[-1,1]$ and $T_{j}\left(B_{m^{k+3} h}(x)\right) \subseteq B_{2 m^{k+2} h}(j)$. Hence by Propositions A.2(a) and A.3, there exists a constant $C>0$ such that

$$
\begin{aligned}
\mu\left(B_{h}\left(T_{i} T_{i^{\prime}} T_{J} T_{j} x\right)\right) & \leq C \cdot c_{i, J} \mu\left(T_{j} B_{m^{k+3} h}(x)\right) \\
& \leqslant C \cdot c_{i, J}\left|\ln \left(2 m^{k+2} h\right)\right|\left(m^{k+2} h\right)^{a_{j}} .
\end{aligned}
$$

By using (A.5), we see that (A.4) is dominated by 


$$
\begin{aligned}
C & \sum_{k=0}^{N} \sum_{|J|=k} h c_{i, J}^{q}\left|\ln \left(2 m^{k+2} h\right)\right|^{q}\left(m^{k+2} h\right)^{q a_{j}} \\
& \leq C|\ln h|^{q} h^{1+q a_{j}} \sum_{k=0}^{N}\left(m^{q a_{j}-(\alpha+\varepsilon)}\right)^{k} m^{(k+2)(\alpha+\varepsilon)}\left(\sum_{|J|=k} c_{i, J}^{q}\right) \\
& \leq C|\ln h|^{q} h^{1+q a_{j}}\left(m^{q a_{j}-(\alpha+\varepsilon)}\right)^{N} \sum_{k=0}^{\infty} m^{(k+2)(\alpha+\varepsilon)}\left(\sum_{|J|=k} c_{i, J}^{q}\right)=o\left(h^{1+\alpha+\varepsilon / 2}\right) .
\end{aligned}
$$

Hence we have the desired estimation for (A.4) and thus for (II). For (I), by using the remark following Proposition 4.3, we have, for $i=1, \ldots, m-2, j=0,1$, $\ldots, m-1, j \neq i^{\prime}$, and $0 \leq x \leq m^{2} h$,

$$
\mu\left(B_{h}\left(T_{i} T_{j} x\right)\right)^{q} \leq C \begin{cases}w_{i-1}^{q} \mu\left(B_{2 m h}(m)\right)^{q}+w_{i}^{q} \mu\left(B_{2 m h}(i+j)\right)^{q} & \text { if } j<i^{\prime} \\ w_{i}^{q} \mu\left(B_{2 m h}(m)\right)^{q}+w_{i+1}^{q} \mu\left(B_{2 m h}\left(j-i^{\prime}\right)\right)^{q} & \text { if } j>i^{\prime},\end{cases}
$$

where $C$ is some constant depending only on $q$. Note that $1 \leq i+j \leq m-2$ and $1 \leq j-i^{\prime} \leq i \leq m-2$. Hence Proposition A.3 implies that $\mu\left(B_{h}\left(T_{i} T_{j} x\right)\right)^{q}$ is of order $o\left(h^{\alpha+\varepsilon / 2}\right)$ as $h \rightarrow 0$. Therefore the integral $\int_{0}^{m^{2} h} \mu\left(B_{h}\left(T_{i} T_{j} x\right)\right)^{q} d x$ is of order $o\left(h^{1+\alpha+\varepsilon / 2}\right)$. This yields the desired estimation for (I). Lastly, in view of Proposition 4.3(b) (together with the remark that follows it) and the first inequality in Proposition A.2(a), we see that (III) and (IV) are dominated by (I) and (II) respectively and this completes the proof.

Recall that

$$
e^{1}(h)=\frac{1}{m^{N+2}} \sum_{i=1}^{m-2} \sum_{|J|=N+1} \int_{0}^{m} \mu\left(B_{h}\left(T_{i} T_{i^{\prime}} T_{J} x\right)\right)^{q} d x .
$$

Lemma A.6. Under the same hypotheses of Lemma A.5, there exists $\varepsilon>0$ such that $e^{1}(h)=o\left(h^{1+\alpha+\varepsilon}\right)$ as $h \rightarrow 0^{+}$.

Proof. Again we need only estimate

$$
\text { (A.7) } \begin{aligned}
\frac{1}{m^{N+2}} & \sum_{|J|=N+1} \int_{0}^{m} \mu\left(B_{h}\left(T_{i} T_{i^{\prime}} T_{J} x\right)\right)^{q} d x \\
& =\frac{1}{m^{N+2}} \sum_{|J|=N} \int_{0}^{m}\left(\mu\left(B_{h}\left(T_{i} T_{i^{\prime}} T_{J} T_{0} x\right)\right)^{q}+\mu\left(B_{h}\left(T_{i} T_{i^{\prime}} T_{J} T_{m-1} x\right)\right)^{q}\right) d x .
\end{aligned}
$$


By symmetry we need only estimate the first sum in (A.7). For $0 \leq x \leq m$ and $|J|=N$, we have

(A.8) $\mu\left(B_{h}\left(T_{i} T_{i^{\prime}} T_{J} T_{0} x\right)\right)^{q} \leq C\left(\mu\left(T_{i} T_{i^{\prime}} T_{J} T_{0}[-1,0]\right)^{q}+\mu\left(T_{i} T_{i^{\prime}} T_{J} T_{0}[0, m]\right)^{q}\right.$

$$
\left.+\mu\left(T_{i} T_{i^{\prime}} T_{J} T_{0}[m, m+1]\right)^{q}\right),
$$

where $C$ can be taken to be $\gamma_{q}^{2}$ with $\gamma_{q}=\max \left\{1,2^{q-1}\right\}$. Let $J=\left(J^{\prime}, m-1\right.$, $0, \ldots, 0),|J|=N$, and $\left|J^{\prime}\right|=k$. Then by applying Propositions A.2(b)(ii) and 4.4(c)(ii) to (A.8), we have

$$
\mu\left(B_{h}\left(T_{i} T_{i^{\prime}} T_{J} T_{0} x\right)\right)^{q} \leq C\left(\left(w_{m-1}^{N-k+1} c_{i, J^{\prime}}\right)^{q}+c_{i, J}^{q}\right) .
$$

Similarly, if $J=J_{0}:=(0, \ldots, 0)$, then by using Proposition A.2(b)(iii) instead, we have

$$
\mu\left(B_{h}\left(T_{i} T_{i^{\prime}} T_{J} T_{0} x\right)\right)^{q} \leq C\left(w_{m-1}^{q(N+2)}+c_{i, J_{0}}^{q}\right) .
$$

Applying (A.9) and (A.10), we see that the first sum in (A.7) is dominated by

$$
\begin{gathered}
\frac{C_{1}}{m^{N+2}}\left(\sum_{k=0}^{N-1} \sum_{\left|J^{\prime}\right|=k}\left(w_{m-1}^{N-k+1} c_{i, J^{\prime}}\right)^{q}+\sum_{\substack{|J|=N \\
J \neq J}} c_{i, J}^{q}\right)+\frac{C_{2}}{m^{N+2}}\left(w_{m-1}^{q(N+2)}+c_{i, J_{0}}^{q}\right) \\
\leq \frac{C_{3}}{m^{N+2}} \sum_{k=0}^{N} \sum_{|J|=k}\left(w_{m-1}^{N-k} c_{i, J}\right)^{q}+\frac{C_{2}}{m^{N+2}} w_{m-1}^{q(N+2)},
\end{gathered}
$$

where $C_{1}, C_{2}, C_{3}$ are constants. Choose $\varepsilon>0$ sufficiently small so that $(q, \alpha+\varepsilon) \in$ $D$ and $\alpha+\varepsilon<q a_{j}$ for all $j$. Then the sum in the expression above is dominated by

$$
\begin{aligned}
& \frac{C}{m^{N+2}} \sum_{k=0}^{N} m^{-(k+2)(\alpha+\varepsilon)} w_{m-1}^{q(N-k)} m^{(k+2)(\alpha+\varepsilon)}\left(\sum_{|J|=k} c_{i, J}^{q}\right) \\
& \leq C \frac{m^{-N q a_{m-1}}}{m^{N+2}} \sum_{k=0}^{N}\left(m^{q a_{m-1}-(\alpha+\varepsilon)}\right)^{k} m^{(k+2)(\alpha+\varepsilon)}\left(\sum_{|J|=k} c_{i, J}^{q}\right) \\
& \left.\quad \quad \quad \text { (since } w_{m-1} \leq m^{-a_{m-1}}\right) \\
& \leq C \frac{m^{-N q a_{m-1}}}{m^{N+2}}\left(m^{q a_{m-1}-(\alpha+\varepsilon)}\right)^{N} \\
& =o\left(h^{1+\alpha+\varepsilon}\right) .
\end{aligned}
$$


Lastly, it can be checked directly that the term $w_{m-1}^{q(N+2)} / m^{N+2}$ is also of order $o\left(h^{1+\alpha+\varepsilon}\right)$, and the proof is complete.

Combining Proposition A.4 and Lemmas A.5 and A.6, we have

Lemma A.7. Suppose $q>0$. Then the equality $F(q, \alpha)=1$ is attained in the region $\left\{(q, \alpha) \in D \mid \alpha<q a_{j}\right.$ for all $\left.j=0,1, \ldots, m\right\}$. Moreover, if $(q, \alpha)$ is in this region, then there exists $\varepsilon>0$ such that $E(h)=o\left(h^{\varepsilon}\right)$ as $h \rightarrow 0^{+}$.

Lemma 4.6 follows by combining this lemma and Proposition A.1.

Acknowledgements. The second author gratefully thanks Professor K. F. Ng for his constant interest and support. He also thanks Professors R. Q. Jia, J. Z. Wang, and D. X. Zhou for some helpful conversations. Part of this research was completed while the second authors was visiting the Department of Mathematics, Cornell University. He would like to thank the department for its hospitality and support.

The first author was partially supported by an RGC Grant from Hong Kong. The second author was partially supported by a postdoctoral fellowship from Hong Kong.

\section{REFERENCES}

[AY] J. C. AleXander \& J. A. Yorke, Fat baker's transformations, Ergodic Theory Dynamical Systems 4 (1984), 1-23.

[AZ] J. C. AleXANDer \& D. Zagier, The entropy of a certain infinitely convolved Bernoulli measure, J. London Math. Soc. 44 (1991), 121-134.

[AP] M. ARBEITER \& N. PATZSCHKE, Random self-similar multifractals, Math. Nachr. 181 (1996), 5-42.

[CM] R. CAWley \& R. D. Mauldin, Multifractal decompositions of Moran fractals, Adv. Math. 92 (1992), 196-236.

[EM] G. A. EDGAR \& R. D. MAULDIN, Multifractal decompositions of digraph recursive fractals, Proc. London Math. Soc. 65 (1992), 604-628.

[F] K. J. Falconer, Techniques in Fractal Geometry, John Wiley \& Sons, Ltd, Chichester, 1997.

[FLN] A.-H. FAN, K.-S. LAU \& S.-M. NGAI, Iterated function systems with overlaps, Asian J. Math., to appear.

[Fe] W. Feller, An Introduction to Probability Theory an its Applications, Vol II, Second edition, John Wiley \& Sons, Inc, New York-London-Sydney, 1971.

[Fen1] D.-J. FENG, The limit Rademacher functions and Bernoulli convolutions associated with Pisot numbers, preprint, 1999.

[Fen2] - On the infinite similarity and multifractal analysis of the Bernoulli convolutions, preprint, 1999. 
[HP] H. G. E. Hentschel \& I. Procaccia, The infinite number of generalized dimensions of fractals and strange attractors, Phys. D 8 (1983), 435-444.

[H] Y. HEURTEAUX, Estimations de la dimension inférieure et de la dimension supérieure des mesures, Ann. Inst. H. Poincaré Probab. Statist. 34 (1998), 309-338.

[Hu] T.-Y. HU, The local dimension of the Bernoulli convolution associated with the golden number, Trans. Amer. Math. Soc. 349, (1997), 2917-2940.

[HL] T.-Y. HU \& K.-S. LAU, Multifractal structure of convolution of the Cantor measure, Adv. in Appl. Math., to appear.

[Hut] J. E. HutCHinsON, Fractals and selfsimilarity, Indiana Univ. Math. J. 30 (1981), 713-747.

[L] S. P. LALLEY, Random series in powers of algebraic integers: Hausdorff dimension of the limit distribution, J. London Math. Soc. 57 (1998), 629-654.

[La1] K.-S. LAU, Fractal measures and mean p-variations, J. Funct. Anal. 108 (1992), 427-457.

[La2] _ Dimension of a family of singular Bernoulli convolutions, J. Funct. Anal. 116 (1993), 335-358.

[LWC] K.-S. LAU, J. WANG \& C.-H. CHU, Vector-valued Choquet-Deny theorem, renewal equation and self-similar measures, Studia Math. 117 (1995), 1-28.

[LN1] K.-S. LAU \& S.-M. NGAI, Multifractal measures and a weak separation condition, Adv. Math. 141, (1999), 45-96.

[LN2],$L^{q}$-spectrum of the Bernoulli convolution associated with the golden ratio, Studia Math. 131 (1998), 225-251.

[LN3],$L^{q}$-spectrum of Bernoulli convolutions associated with P.V. numbers, Osaka J. Math. 36 (1999), 993-1010.

[LP] F. Ledrappier \& A. PORZIO, A dimension formula for Bernoulli convolutions, J. Statist. Phys. 76 (1994), 1307-1327.

[N1] S.-M. NGAI, Multifractal decomposition for a family of overlapping self-similar measures, In: Fractal Frontiers, (M. M. Novak \& T. G. Dewey eds.), World Sci. Publishing, River Edge, NJ, 1997, pp. 151-161.

[N2] - A dimension result arising from the $L^{q}$-spectrum of a measure, Proc. Amer. Math. Soc. 125 (1997), 2943-2951.

[O1] L. OlSEN, A multifractal formalism, Adv. Math. 116 (1995), 82-196.

[O2] Dimension inequalities of multifractal Hausdorff measures and multifractal packing measures, Math. Scand. 86 (2000), 109-129.

[P] A. PORZIO, On the regularity of the multifractal spectrum of Bernoulli convolutions, J. Statist. Phys. 91 (1998), 17-29.

[R] R. RIEDI, An improved multifractal formalism and self-similar measures, J. Math. Anal. Appl. 189 (1995), 462-490.

[Ro] H. L. RoYden, Real Analysis (Third edition), Macmillan Publishing Company, New York, 1988.

[S] A. SCHIEF, Separation properties for self-similar sets, Proc. Amer. Math. Soc. 122 (1994), 111-115. 
[St] R. S. STRICHARTZ, Self-similar measures and their Fourier transforms III, Indiana Univ. Math. J. 42 (1993), 367-411.

[STZ] R. S. STRICHARTZ, A. TAYLOR \& T. ZHANG, Densities of self-similar measures on the line, Experiment. Math. 4 (1995), 101-128.

KA-SING LAU

Department of Mathematics

The Chinese University of Hong Kong

Shatin, NT, HONG KONG

kslau@math.cuhk.edu.hk

\author{
SZE-MAN NGAI \\ School of Mathematics \\ Georgia Institute of Technology \\ Atlanta, Georgia 30332, U. S. A. \\ CURRENT ADDRESS: \\ Dept. of Mathematics and Computer Science \\ Georgia Southern University \\ Statesboro, Georgia 30460, U. S. A.
}

SUBJECT CLASSIFICATION: primary 28A80; secondary 28 A78.

KEYWORDS: self-similar measure, second-order identity, multifractal formalism, $L^{q}$-spectrum, dimension spectrum.

Submitted: April 5th, 1999, revised: March 9th, 2000. 